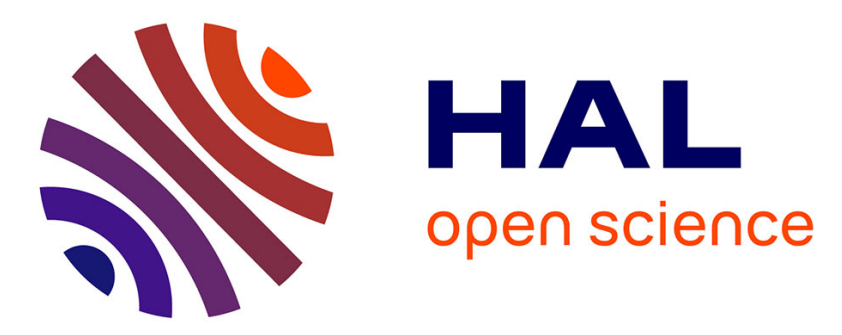

\title{
A homogenization approach for assessing the yield strength properties of stone column reinforced soils
}

Ghazi Hassen, Maxime Gueguin, Patrick de Buhan

\section{To cite this version:}

Ghazi Hassen, Maxime Gueguin, Patrick de Buhan. A homogenization approach for assessing the yield strength properties of stone column reinforced soils. European Journal of Mechanics - A/Solids, 2013, 37, pp.266-280. 10.1016/j.euromechsol.2012.07.003 . hal-00833484

\section{HAL Id: hal-00833484 \\ https://hal-enpc.archives-ouvertes.fr/hal-00833484}

Submitted on 12 Jun 2013

HAL is a multi-disciplinary open access archive for the deposit and dissemination of scientific research documents, whether they are published or not. The documents may come from teaching and research institutions in France or abroad, or from public or private research centers.
L'archive ouverte pluridisciplinaire HAL, est destinée au dépôt et à la diffusion de documents scientifiques de niveau recherche, publiés ou non, émanant des établissements d'enseignement et de recherche français ou étrangers, des laboratoires publics ou privés. 


\title{
A homogenization approach for assessing the yield strength properties of stone column reinforced soils
}

\author{
Ghazi HASSEN, Maxime GUEGUIN and Patrick de BUHAN ${ }^{(*)}$ \\ Université Paris-Est, Laboratoire Navier, Ecole des Ponts ParisTech-IFSTTAR-CNRS, \\ 6 et 8 av. B. Pascal, 77455 Marne-la-Vallée, France \\ ${ }^{(*)}$ Corresponding author: patrick.debuhan@enpc.fr
}

\begin{abstract}
The macroscopic strength properties of a purely cohesive soil reinforced by a periodic distribution of "stone columns" made of a highly frictional granular material are investigated in a rigorous way on the basis of the yield design homogenization approach. Starting from a first crude lower bound approximation to the macroscopic strength criterion of the stone column reinforced soil, a much more accurate failure surface is then drawn in the space of stresses as a result of a series of numerical elastoplastic simulations performed on the reinforced soil unit cell subject to radial strain controlled loading paths. The anisotropic characteristics of the so obtained original criterion are then highlighted by means of its representation in the Mohr plane attached to any oriented facet. The paper concludes with a first illustrative implementation of the method on the derivation of an upper bound estimate for the ultimate bearing capacity of a stone column reinforced foundation.
\end{abstract}

Key words: yield design; periodic homogenization; macroscopic strength criterion; reinforced soil; stone column; load bearing capacity. 


\section{Introduction}

Many attempts have been made over at least the past thirty years to predict the overall strength properties of fiber or inclusion-reinforced composite materials from the knowledge of the strength characteristics of their individual components (matrix and reinforcement) along with such key parameters as the reinforcement volume fraction. Referring more specifically to the limit analysis or yield design method applied to periodic media, the fundamentals of which have been laid by Suquet (1985) in a general framework or de Buhan (1986) in the context of reinforced soil mechanics, the macroscopic strength condition of such composites are derived from the solution to a yield design boundary value problem relative to the unit periodic cell.

In the particular situation when the fiber volume fraction is small, whereas the reinforcing material (metal or concrete) exhibits considerably higher strength characteristics than those of the matrix (soil in the case of inclusion-reinforced soils), a quite simplified, but exact, formulation of the macroscopic strength condition may be obtained: see for instance McLaughlin (1972), Majamdar and McLaughlin (1975), de Buhan and Salençon (1987) or de Buhan and Taliercio (1991). As regards engineering applications in the field of geomechanics, this criterion has proved particularly convenient for describing the global strength anisotropy of reinforced earth and thus provide a rational basis for stability analyses of structures: Sawicki (1983), de Buhan et al. (1989), Sawicki and Lesniewska (1989), di Prisco and Nova (1993), Abdi et al. (1994), Michalowski and Zhao (1995), Michalowski (1997).

Unfortunately, this simplified criterion is not appropriate to describe the macroscopic yield strength of soft foundation soils reinforced by cylindrical inclusions or columns, since the two above mentioned conditions are not satisfied. Indeed, according to this type of soil improvement technique, the volume fraction of the columns (also called substitution factor) may range between $10 \%$ to $40 \%$, while at the same time, the strength properties of the column material are higher, but remain of the same order as those of the soil. Considering for instance a soft clayey foundation soil, two subcategories of reinforcement techniques by columns may be envisaged, depending on the kind of column material to be used.

The so called "lime column" reinforcement technique (Broms, 1982) consists in mixing the weak soil mass with a given percentage of lime or lime-cement, thus providing an important increase of the soil initial shear strength (up to 20 times) along with a relatively small friction angle, which can be neglected as a first approximation. It this case, where both constituents are 
modeled as purely cohesive materials (i.e. obey Tresca or von Mises conditions), the macroscopic strength criterion is of the purely cohesive, but anisotropic kind, with the column orientation as symmetry axis. A fairly accurate closed form expression of this criterion can be derived and then incorporated into yield design calculations of reinforced soil structures with no particular difficulty (Jellali et al., 2005, 2011).

The second main category is the "stone column" technique, where the reinforcing material is a vibrocompacted granular material or ballast exhibiting high frictional properties with a negligible cohesion (Priebe, 1995). The strength of the column material is adequately described by a MohrCoulomb (or Drucker-Prager) criterion and the question may then arise as to how the soil is actually strengthened by the stone columns. This problem is illustrated in Figure 1, where the Tresca (respectively Mohr-Coulomb) criterion adopted for the soil (respectively column material) is represented as an intrinsic curve drawn in the Mohr plane. As can be immediately seen from this Figure, the purely frictional column material is more resistant than the initial purely cohesive soil for large compressive normal stresses, but offers for instance no resistance at all to tensile stresses.

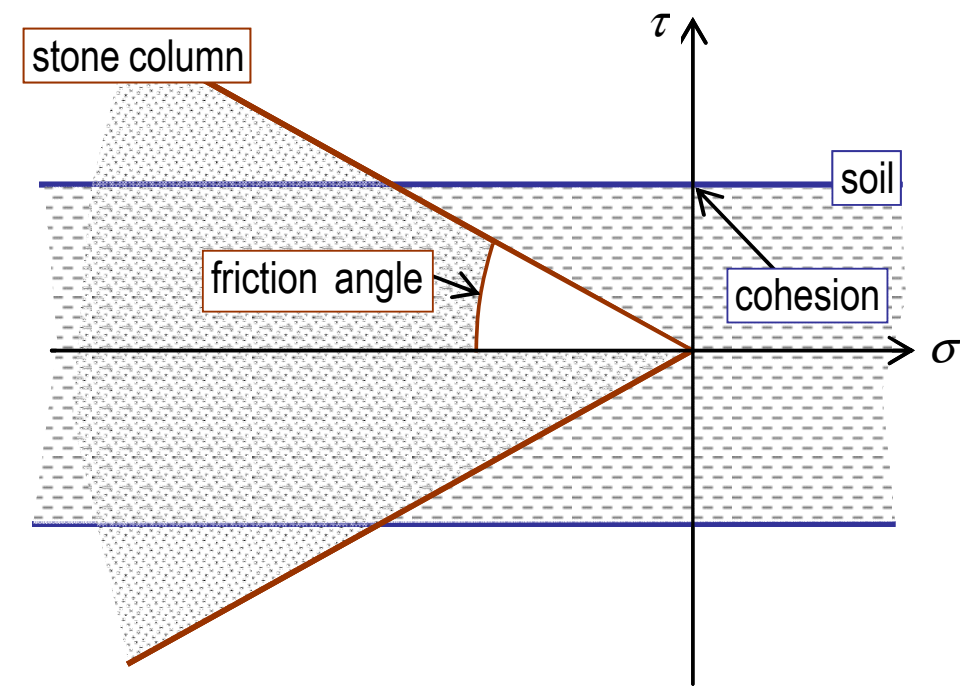

Figure 1. Representation of the soil and stone column strength criteria in the Mohr plane

A sufficiently accurate and reliable knowledge of the macroscopic strength criterion is therefore needed for assessing the actual reinforcing effect to be expected from installing frictional columnar inclusions into the purely cohesive soft soil. The present contribution is devoted to this task, striving to derive in a rigorous way the macroscopic strength condition on the basis of the yield design homogenization method for periodic media. 


\section{Stability analysis of stone column-reinforced soil structures: a challenging issue}

\subsection{Yield strength properties of soil and column materials}

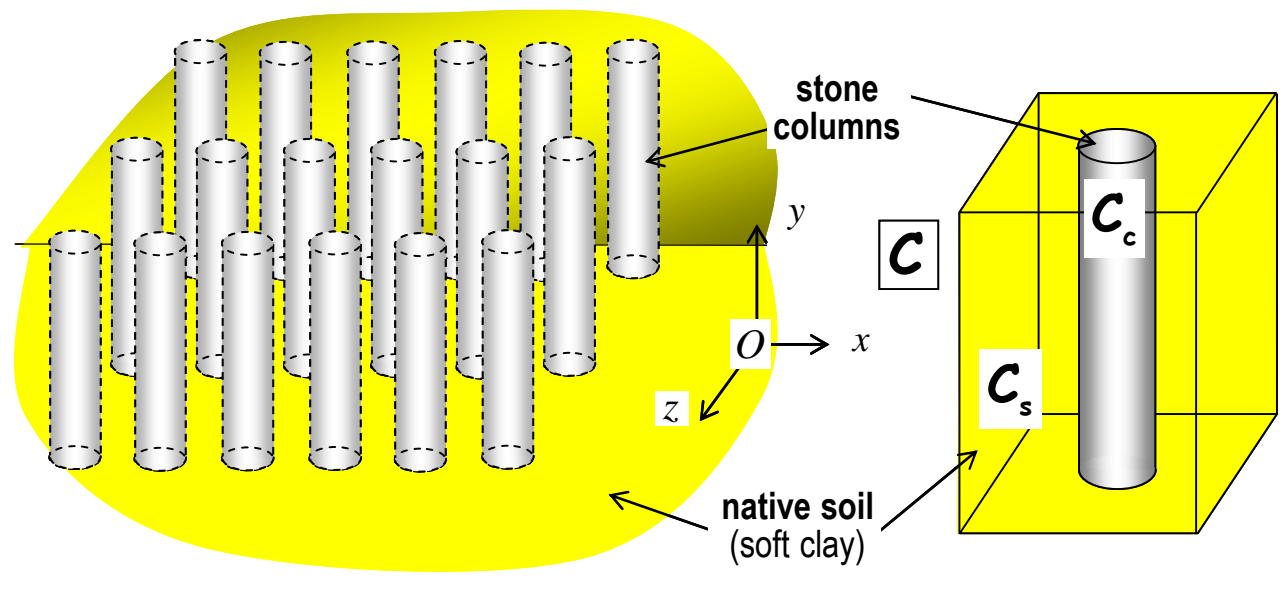

(a)

(b)

Figure 2. (a) stone column reinforced soil and $(b)$ representative unit cell

A stone column-reinforced soil as sketched in Figure 2 may be perceived as a "geo-composite" material, made of a regular array of cylindrical columnar inclusions embedded into the soil mass. The strength properties of each component of such a composite may be described as follows.

a) The native soil is generally a purely cohesive soft clay, the strength condition of which will be described by a von Mises yield condition of the form:

$$
f^{s}(\underline{\underline{\sigma}})=\sqrt{1 / 2 \underline{\underline{s}}: \underline{\underline{s}}}-k
$$

where $\underline{\underline{\underline{s}}}$ denotes the deviatoric stress and $k$ yield strength under pure shear conditions.

b) Likewise, the column constituent material is a purely frictional granular soil or ballast obeying a Drucker-Prager strength criterion of the form:

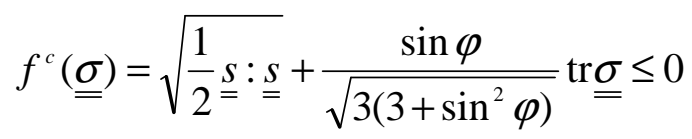

where $\varphi$ represents the friction angle. It is worth noting that the above formulation has been chosen in such a way that the Drucker-Prager criterion coincides, under plane strain conditions, with the classical Mohr-Coulomb criterion associated with the same friction angle $\varphi$. 


\subsection{Implementation of the upper bound kinematic approach: a tricky problem}

The stability analysis of stone column reinforced structures can be performed in the context of the yield design (or limit analysis) framework on the basis of the previously introduced strength conditions adopted for the soil and the columns, respectively. According to this theory (see Salençon (1990), for more details), the stability of such a structure is ensured if one can exhibit a stress field $\underline{\underline{\sigma}}$ in equilibrium with the loading (statically admissible), while satisfying the strength condition of the different constituents at any point:

$$
\text { Stability } \Leftrightarrow\left\{\begin{array}{l}
\exists \underline{\underline{\sigma}} \text { statically admissible } \\
f^{s}(\underline{\underline{\sigma}}) \leq 0 \text { in the soil } \\
f^{c}(\underline{\underline{\sigma})} \leq 0 \text { in the columns }
\end{array}\right.
$$

The dualisation of the equilibrium conditions by means of the virtual work principle leads to the much more frequently employed upper bound kinematic method, as opposed to the lower bound static one derived from definition (3). This method is based on considering virtual velocity fields ("failure mechanisms") such as those displayed for instance in Figure 3, where rigid body moving blocks, separated by velocity jump surfaces are involved. The extreme difficulty to perform the upper bound kinematic approach will now be explained on this particular class of failure mechanisms.

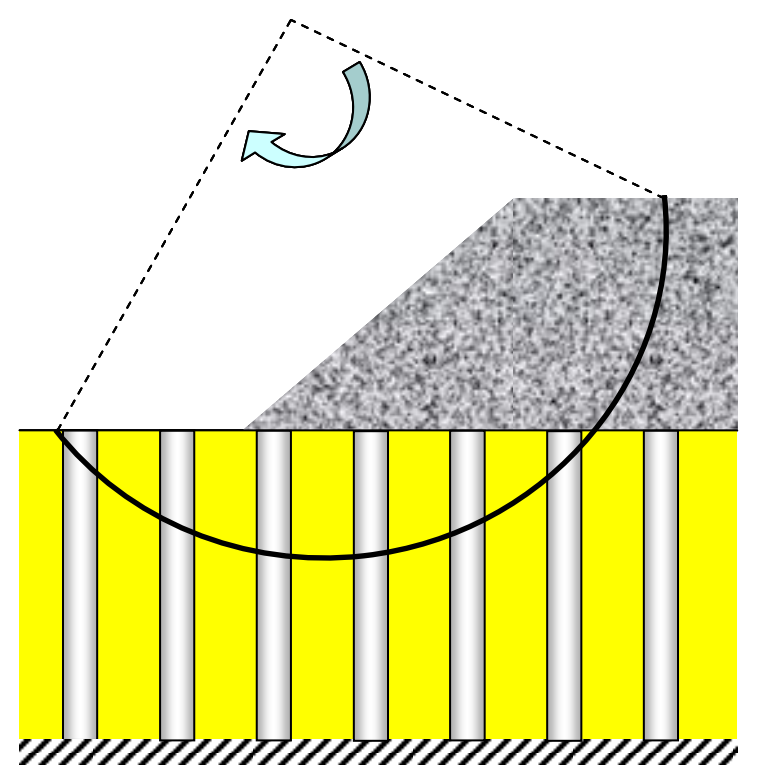

(a)

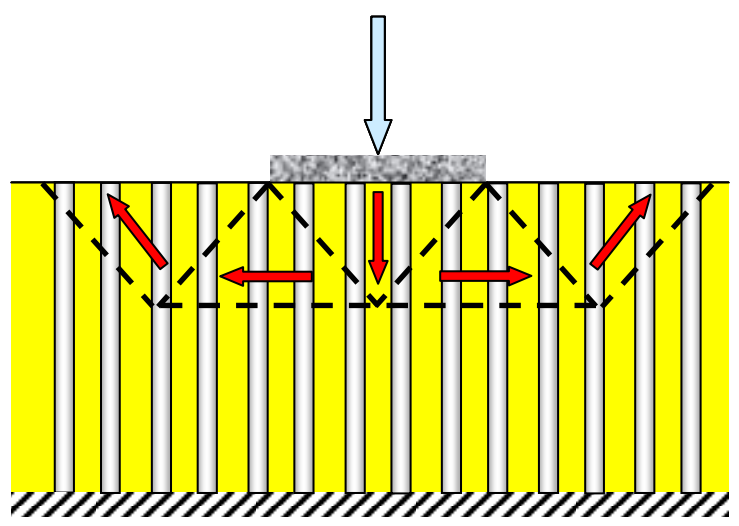

(b)

Figure 3: Yield design of stone column reinforced soil structures making use of the upper bound kinematic method: $(a)$ rotational and $(b)$ translational failure mechanisms 
Indeed, a key ingredient to the kinematic method of yield design is the so-called "maximum resisting work", defined as follows in the case of mechanisms based on discontinuity surfaces:

$$
\left.W_{m r}(\underline{U})=\int_{\Sigma} \pi(\underline{n} ; \underline{U}]\right) \mathrm{d} \Sigma
$$

where $[\underline{U}]$ represents the velocity jump when crossing the discontinuity surface $\Sigma$ along its unit normal $\underline{n}$. According to the kinematic approach of yield design, a necessary condition for the structure to remain stable in the sense defined by (3), is that the work developed by the external forces (loading) in any velocity field remains lower than or equal to the maximum resisting work (4). The expression of the support function $\pi$ appearing in (4) is completely different depending on whether the velocity jump is located in the soil or in the column.

a) Since the native soil is purely cohesive, the velocity jump must be tangential to the discontinuity surface (figure $4(a)$ ) leading to the classical following expression for the corresponding support function:

$$
\pi^{s}(\underline{n},[\underline{U}])=\left\{\begin{array}{lc}
k|[\underline{U}]| & \text { if }[\underline{U}] \cdot \underline{n}=0 \\
+\infty & \text { otherwise }
\end{array}\right.
$$

b) As regards a velocity jump located in the purely frictional column material, this support function becomes:

$$
\pi^{c}(\underline{n},[\underline{U}])=\left\{\begin{array}{lll}
0 & \text { if } & {[\underline{U}] \cdot \underline{n} \geq \mid[\underline{U}] \sin \varphi} \\
+\infty & \text { otherwise }
\end{array}\right.
$$

which means that the velocity jump must be inclined at an angle larger than $\varphi$ with respect to the discontinuity surface, as shown in Figure 4(b).

Velocity jumps complying with the kinematic conditions contained in (5) and (6), for which the support functions take finite values, are said to be "relevant" (Salençon, 1990). This terminology simply means that "irrelevant" velocity jumps would produce infinite values for the support function and thus for the maximum resisting work (4), leading to infinite upper bound values, that is providing no information as regards the stability analysis of the structure. Referring to the more conventional plastic limit analysis, where the strength criterion is a plastic yield condition, such purely mathematical conditions are perfectly equivalent to saying that the velocity jumps and associated failure mechanisms are "plastically admissible". 
Let us now consider a velocity jump surface $\Sigma$ running through the composite reinforced soil as sketched in Figure 4(c). It follows from (5) that the velocity jump has to be tangential to the surface at any point of its intersection $\Sigma_{s}$ with the soil, while, according to (6), it should on the contrary make an angle at least equal to $\varphi$, at any point of its intersection $\Sigma_{c}$ with the column. This is obviously feasible for a soil reinforced by trenches or layers perpendicular to the plane of motion, the transverse cross section of the discontinuity surface being a piecewise linear or "broken" line as drawn in Figure 4(d), constructed in such a way that the velocity jumps remain relevant in the soil and the reinforcing trench.

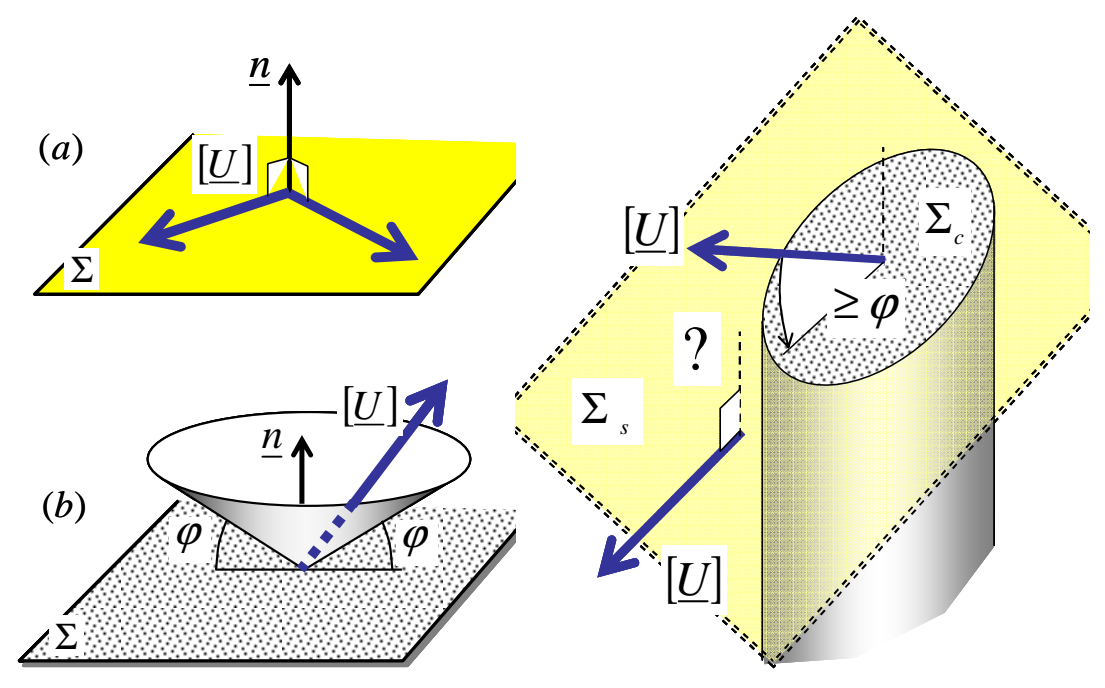

(c)

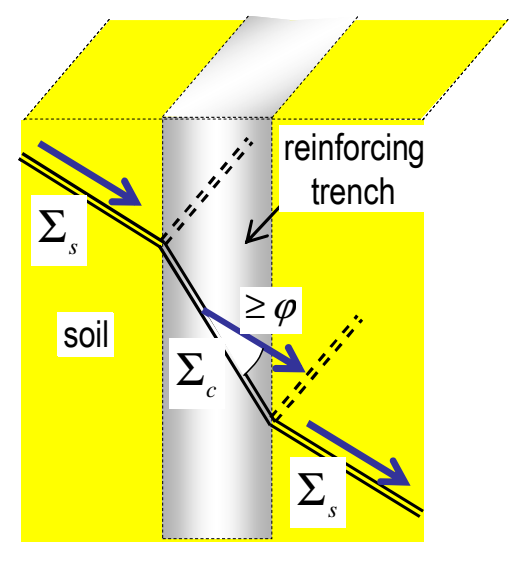

$(d)$

Figure 4. Relevant velocity jump surfaces: $(a)$ in the soil; $(b)$ the reinforcement; $(c)$ a stone column reinforced soil; $(d)$ a trench reinforced soil

On the other hand, in the case of a stone column reinforced soil, the construction of such relevant failure surfaces and associated mechanisms proves hardly feasible, if not impossible: to the Authors' knowledge, no such relevant mechanism have been exhibited so far. It is primarily due to the three-dimensional configuration of the stone column reinforcement as suggested by Figure 4(c). This dead end clearly undermines the very use of any upper bound kinematic approach for analyzing the stability of this kind of reinforced soil structure, in the rigorous framework of the yield design theory. As it will be seen now, this major difficulty can be overcome, by resorting to the yield design homogenization method, where the composite reinforced soil will be treated as homogeneous anisotropic medium. 


\section{Outline of the periodic homogenization method}

\subsection{Macroscopic strength condition}

The basic features of the periodic homogenization method aimed at solving yield design problems, such as that formulated in the previous section, are briefly outlined in this section. A detailed presentation may be found in Suquet (1985), de Buhan (1986), de Buhan et al. (1987), de Buhan and Taliercio (1991) or more recently Jellali et al. (2005, 2011) focusing on column reinforced soils.

Owing to the fact that the reinforcing columns are distributed throughout the soil mass following a regular pattern (Figure 2(a)), the reinforced ground may be perceived as a periodic composite material, the morphology of which is entirely described by a unit cell $\boldsymbol{C}$ of side $s$ (spacing between two neighbouring columns). This unit cell contains one single reinforcing column of radius $\rho$ surrounded by the native soil (Figure 2(b)). The reinforcement volume fraction (also called replacement ratio) is classically defined as the ratio between the volume occupied by the column and the volume of the unit cell:

$$
\eta=\frac{\pi \rho^{2}}{s^{2}}
$$

In practice, the value of this parameter ranges from the $10 \%$ and $40 \%$.

The homogenization method stems from the intuitive idea that, in the formulation of a yield design problem, the composite reinforced soil can be replaced by an equivalent homogeneous medium, the strength properties of which being specified by means of a macroscopic strength criterion. An important result of this method states that this macroscopic can be derived from solving a yield design problem attached to the unit cell $\boldsymbol{C}$ and called auxiliary problem. More specifically, the macroscopic strength criterion is defined as follows:

$$
F(\underline{\underline{\Sigma}}) \leq 0 \Leftrightarrow\left\{\begin{array}{c}
\underline{\underline{\sigma}} \text { statically admissible with } \underline{\underline{\Sigma}} \\
\forall \underline{\xi} \in \mathcal{C}_{\alpha} f^{\alpha}\left(\underline{\underline{\sigma}}^{\alpha}(\underline{\xi})\right) \leq 0
\end{array}\right.
$$

where $C_{\alpha}$ is the unit cell sub-domain occupied by constituent $\alpha$ (c for column or $s$ for soil) and $f^{\alpha}($.) its yield strength function given by either (1) or (2). A stress field $\underline{\underline{\sigma}}$ is statically admissible with a macroscopic stress $\underline{\underline{\underline{\Sigma}}}$ if it complies with the following conditions:

- $\underline{\underline{\sigma}}$ is in equilibrium with no body forces: 


$$
\operatorname{div} \underline{\underline{\sigma}}=0
$$

- the stress vector remains continuous across any possible discontinuity surfaces of the stress field:

$$
[\underline{\underline{\sigma}}] \underline{n}=0
$$

where $[\underline{\underline{\sigma}}]$ denotes the jump of $\underline{\underline{\sigma}}$ across such a surface following its unit normal $\underline{n}$.

- $\underline{\underline{\sigma}} \underline{\underline{n}}$ is anti-periodic, which means that it takes opposite values at any couple of points located on the opposite sides of the unit cell.

$\square \underline{\underline{\Sigma}}$ is equal to the volume average of $\underline{\underline{\sigma}}$ over the unit cell:

$$
\underline{\underline{\Sigma}}=\frac{1}{|C|} \int_{C} \underline{\underline{\sigma}} \mathrm{d} C=\langle\underline{\underline{\sigma}}\rangle
$$

\subsection{Lower bound approximation to the plane strain macroscopic strength condition}

A first lower bound approximation to the strength domain may be obtained from performing the static approach of yield design, that is implementing definition (8) using piecewise homogeneous stress field, as it has previously been done in Jellali et al. (2005) for purely cohesive reinforcing columns or by Jellali et al. (2007) in the case of a column material obeying a MohrCoulomb condition.

Looking forward to performing the stability analysis of plane strain problems, our analysis is now focused on the determination of the macroscopic strength criterion subject to plane strain conditions in the $O x y$-plane (Figure 2). Denoting by $\underset{\underline{\tilde{\Sigma}}}{\underline{ }}$ the two-dimensional tensor formed by the components of a macroscopic stress tensor $\underline{\underline{\Sigma}}$ in the $O x y$-plane:

$$
\underline{\underline{\tilde{\Sigma}}}=\Sigma_{i j} \underline{e}_{i} \otimes \underline{e}_{j}, \quad i, j=x, y
$$

the two-dimensional plane strain macroscopic condition associated with the three-dimensional one (8) writes:

$$
\tilde{F}(\underline{\underline{\tilde{\Sigma}}})=\min _{\Sigma_{z z}}\left\{F\left(\underline{\underline{\tilde{\Sigma}}}+\Sigma_{z z} \underline{e}_{z} \otimes \underline{e}_{z}\right)\right\} \leq 0
$$

The following class of piecewise constant stress fields is now considered, defined as: 


$$
\underline{\underline{\sigma}}^{s}=\left(\begin{array}{ccc}
\Sigma_{x x} & \Sigma_{x y} & 0 \\
\Sigma_{x y} & \sigma_{y y}^{s} & 0 \\
0 & 0 & \Sigma_{z z}
\end{array}\right)
$$

in the sub-domain $\boldsymbol{C}_{\boldsymbol{s}}$ of the unit cell occupied by the soil, and:

$$
\underline{\underline{\sigma}}^{c}=\left(\begin{array}{ccc}
\Sigma_{x x} & \Sigma_{x y} & 0 \\
\Sigma_{x y} & \sigma_{y y}^{c} & 0 \\
0 & 0 & \Sigma_{z z}
\end{array}\right)
$$

in the column $C_{c}$

It can be easily verified that any such stress field is statically admissible (in the sense specified in the previous section 3.1.) with the following macroscopic state of stress:

$$
\underline{\underline{\Sigma}}=\Sigma_{x x} \underline{e}_{x} \otimes \underline{e}_{x}+\sum_{y y} \underline{e}_{y} \otimes \underline{e}_{y}+\Sigma_{z z} \underline{e}_{z} \otimes \underline{e}_{z}+\sum_{x y}\left(\underline{e}_{x} \otimes \underline{e}_{y}+\underline{e}_{y} \otimes \underline{e}_{x}\right)
$$

where, on account of (11):

$$
\Sigma_{y y}=\left\langle\sigma_{y y}\right\rangle=\eta \sigma_{y y}^{c}+(1-\eta) \sigma_{y y}^{s}
$$

For the particular states of stress defined by (14) and (15), the strength conditions of the soil (1) and column material (2) may be put in the following form:

$$
\alpha=s, c: f^{\alpha}\left(\underline{\underline{\sigma}}^{\alpha}\right) \leq 0 \Leftrightarrow \sigma_{y y}^{\alpha-}\left(\Sigma_{x x}, \Sigma_{x y}, \Sigma_{z z}\right) \leq \sigma_{y y}^{\alpha} \leq \sigma_{y y}^{\alpha+}\left(\Sigma_{x x}, \Sigma_{x y}, \Sigma_{z z}\right)
$$

so that on account of (17):

$$
(1-\eta) \sigma_{y y}^{s-}+\eta \sigma_{y y}^{c-} \leq \Sigma_{y y} \leq(1-\eta) \sigma_{y y}^{s+}+\eta \sigma_{y y}^{c+}
$$

Consequently the set of stresses $\left(\Sigma_{x x}, \Sigma_{x y}, \Sigma_{y y}\right)$ belonging to the segment:

$$
\left.\left[\Sigma_{y y}^{-}, \Sigma_{y y}^{+}\right] \text {with } \mid \begin{array}{l}
\Sigma_{y y}^{+}\left(\Sigma_{x x}, \Sigma_{x y}\right)=\max _{\Sigma_{z z}}\left\{(1-\eta) \sigma_{y y}^{s+}+\eta \sigma_{y y}^{c+}\right. \\
\Sigma_{y y}^{-}\left(\Sigma_{x x}, \Sigma_{x y}\right)=\min _{\Sigma_{z z}}\left\{(1-\eta) \sigma_{y y}^{s-}+\eta \sigma_{y y}^{c-}\right.
\end{array}\right\}
$$

will obviously satisfy the macroscopic strength condition. The above optimization procedures are carried out numerically.

As an illustrative example, the corresponding yield strength surface has been drawn in the space of non dimensional macroscopic stresses $\left(\Sigma_{x x} / k, \Sigma_{x y} / k, \Sigma_{y y} / k\right)$ for the following typical values: 


$$
\rho=0.3 s \rightarrow \eta=28 \% \quad ; \varphi=35^{\circ}
$$

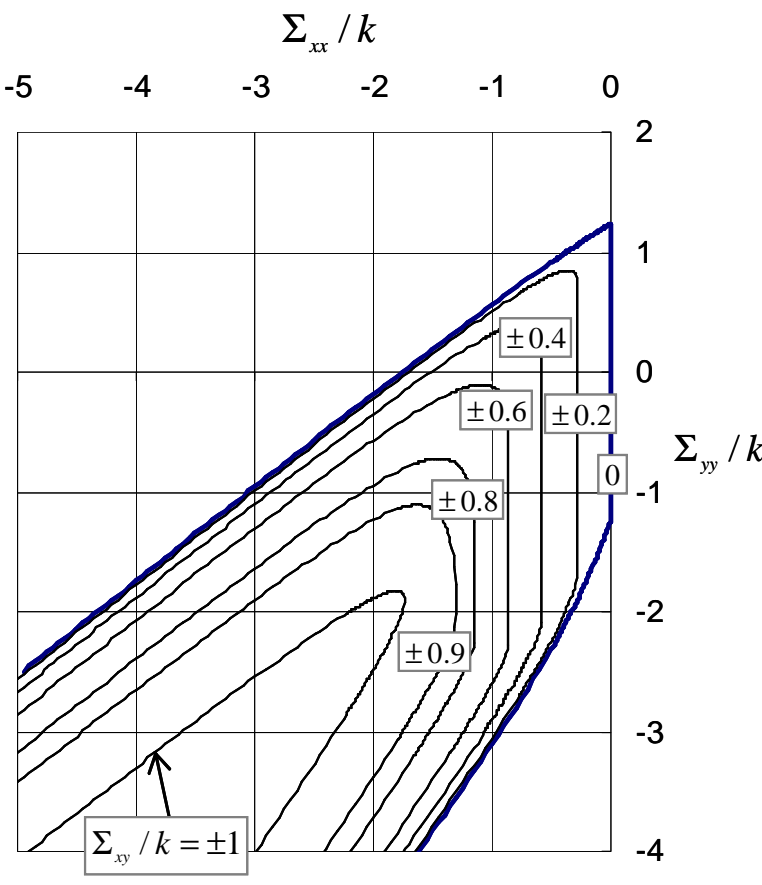

(a)

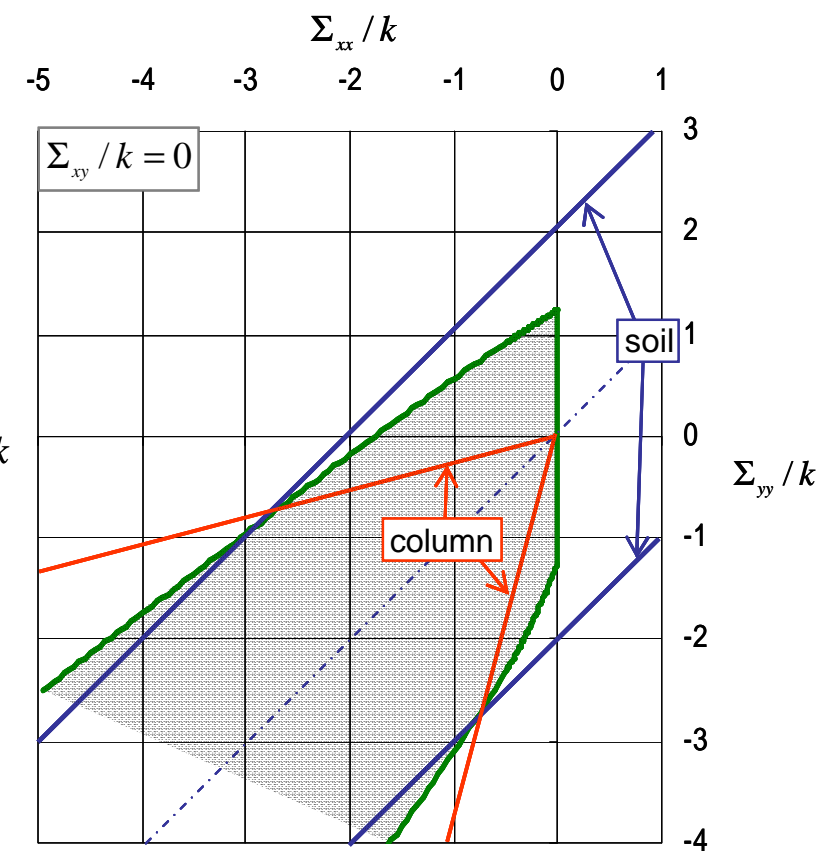

$(b)$

Figure 5: Lower bound estimate for the macroscopic strength domain under plane strain conditions: representation in the $\left(\Sigma_{x x}, \Sigma_{y y}, \Sigma_{x y}\right)$-space.

Figure 5(a) pictures such a surface, in the form of its cross sections by planes of constant non dimensional shear stress $\Sigma_{x y} / k$ varying between -1 and 1 . Figure $5(b)$ displays the particular cross section obtained for zero shear stress (shaded area), as well as those corresponding to the soil and the column material.

\subsection{Representation in the Mohr plane}

Let us consider a facet in the homogenized reinforced soil, with outwards unit normal $\underline{n}$ oriented at an angle $\alpha$ with respect to the direction of reinforcement $O y$ (Figure 6). The normal $\Sigma$ and shear $T$ components generated on this facet by a macroscopic stress state $\underline{\underline{\Sigma}}$ are given by:

$$
\Sigma=\underline{n} \cdot \underline{\underline{\sum} .} \underline{\underline{n}}, \quad T=\underline{t} . \underline{\underline{\Sigma}} \cdot \underline{\underline{n}}
$$

where $\underline{t}=\underline{e}_{z} \wedge \underline{n}$. An alternative, and particularly illustrative, representation of the above lower bound estimate of the macroscopic condition consists in determining for any given oriented facet, the convex envelope of the allowable stress vectors acting upon this facet, defined as: 


$$
G^{L B}(\alpha)=\left\{\left(\Sigma_{n}, T\right) ; F^{L B}(\underline{\underline{\Sigma}}) \leq 0\right\}
$$

where $F^{L B}($.$) is the yield strength function associated with the previously determined lower bound$ approximation.

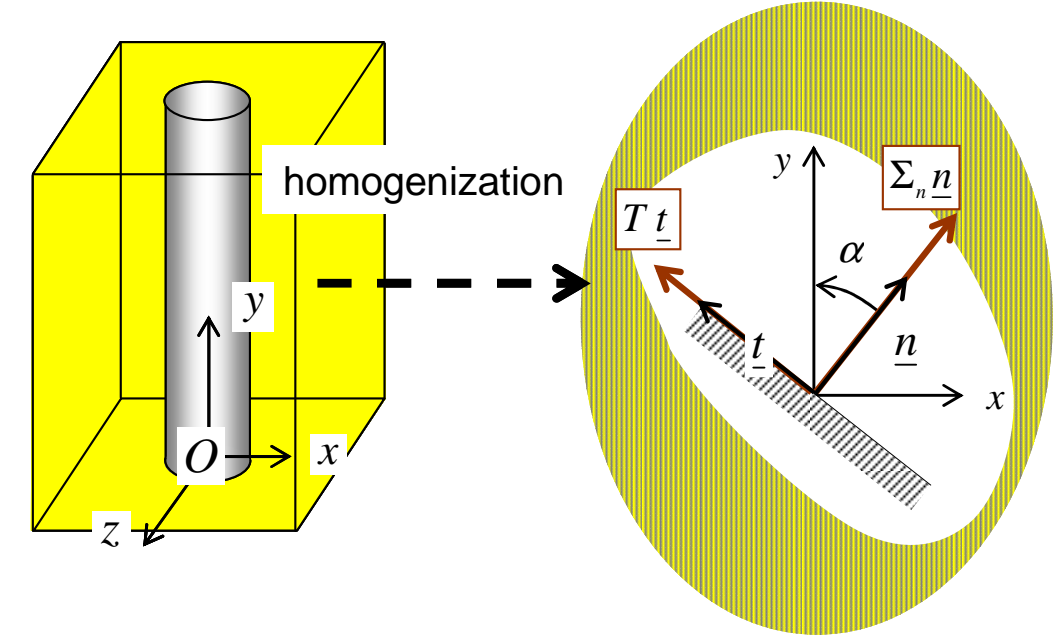

Figure 6. Normal and shear macroscopic stress components acting upon an oriented facet in the homogenized reinforced soil

The results of the analysis are presented in Figure 7, showing the strength domain $\Theta^{A B}(\alpha)$ for differently oriented facets, the axes of the Mohr plane being put in non dimensional form. Figure 8 provides another representation, where the different strength domains have been gathered in the same Mohr plane.

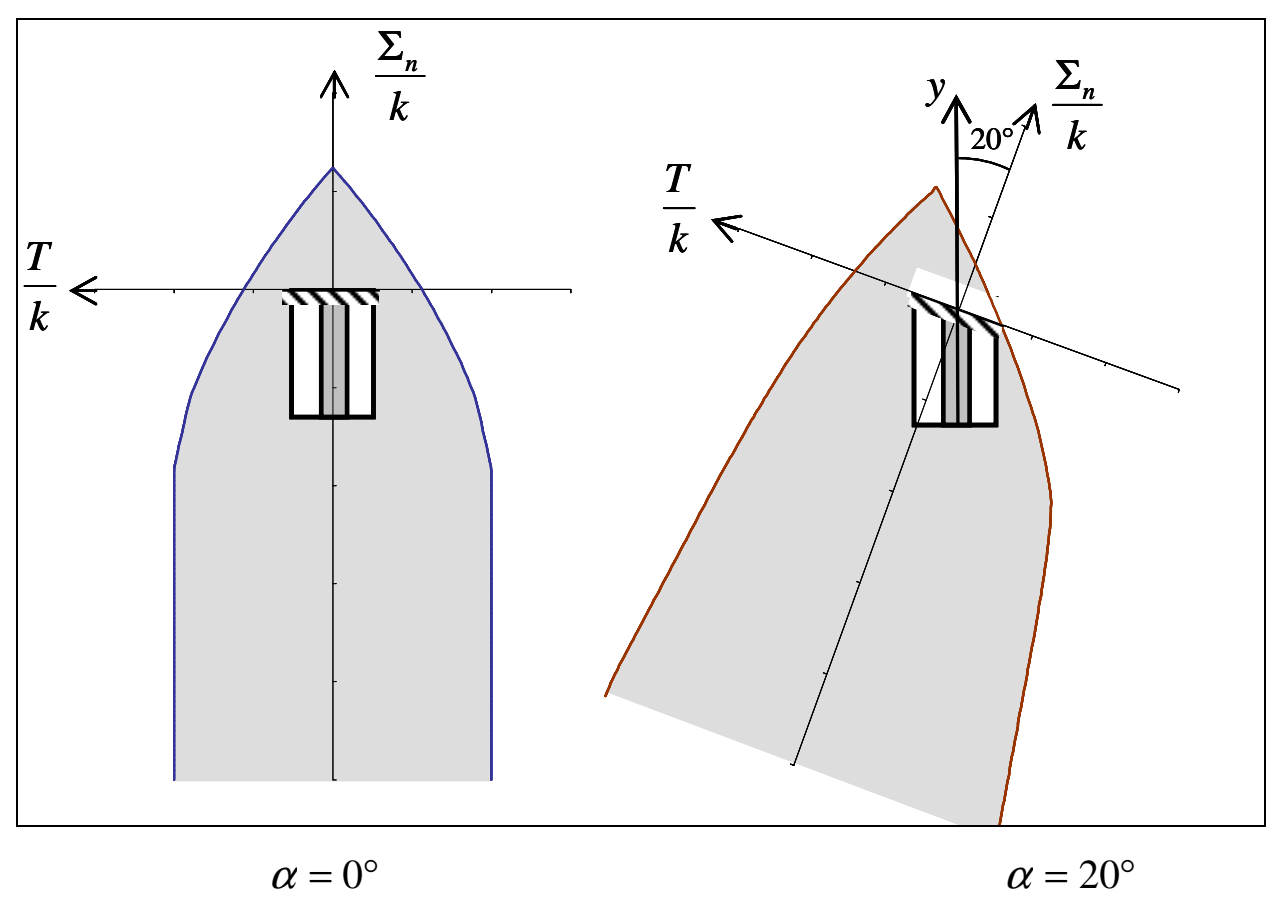



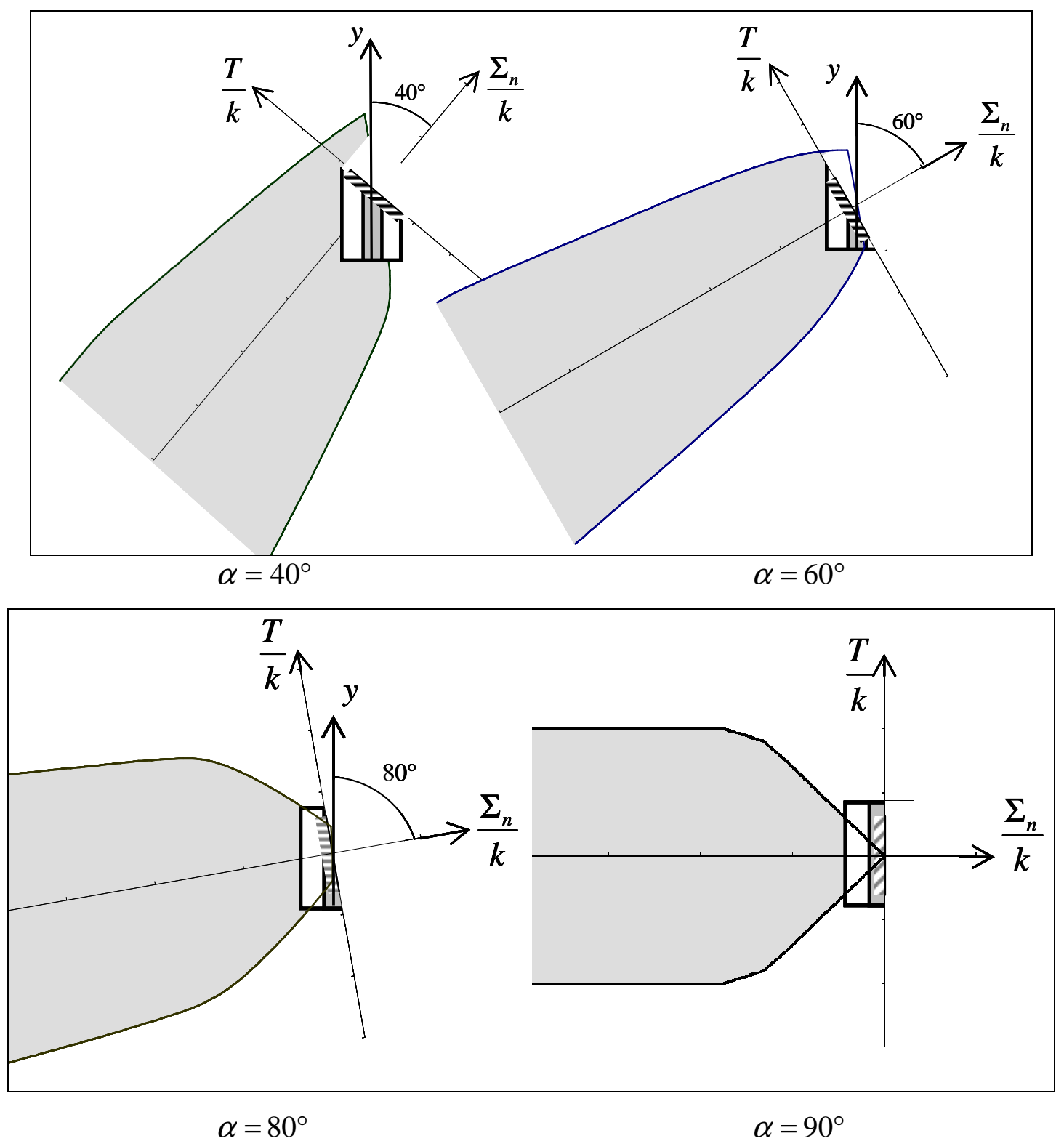

Figure 7: Representation in the Mohr plane of the lower bound approximation to the macroscopic strength condition for different facet orientations

These results deserve two comments.

a) The fact that the strength domain $G^{B}$ in the Mohr plane strongly depends on the facet orientation $\alpha$, as it is quite apparent from both Figures 7 and 8, should be clearly attributed to the anisotropic strength characteristics of the homogenized reinforced soil, due to the preferential orientation of the reinforcing columns. The macroscopic strength condition (or at least its lower bound approximation) is therefore of a general anisotropic cohesive-frictional kind, that is in no way reducible to a classical Mohr-Coulomb criterion or even to any "intrinsic curve" type criterion. 


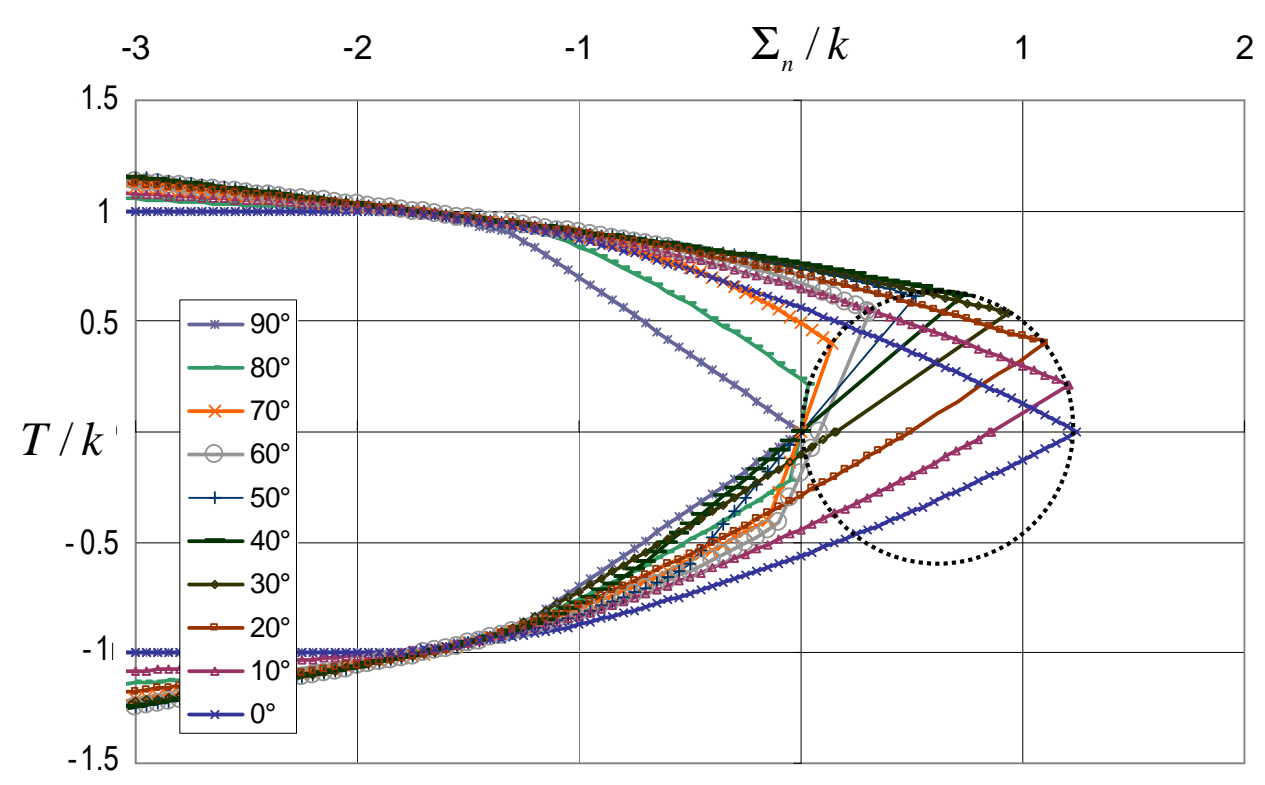

Figure 8. Representation of the yield strength curves determined from the lower bound approximation to the macroscopic strength condition

b) The boundary line of each domain $G^{A B}$ exhibits an angular vertex lying on a circle in the Mohr plane (dashed circle in Figure 8). Indeed, each of these vertices corresponds to the end point of the stress vector generated on the inclined facet by the following particular macroscopic stress:

$$
\underline{\underline{\Sigma}}=\eta{\underline{\underline{\sigma^{c}}}}^{c}(1-\eta){\underline{\underline{\sigma^{s}}}}^{s} \text { with } \mid \begin{aligned}
& {\underline{\underline{\sigma^{c}}}}^{c}=0 \rightarrow f^{c}\left({\underline{\underline{\sigma^{c}}}}^{c}\right)=0 \\
& \underline{\underline{\underline{\sigma}}}^{s}= \pm k \sqrt{3} \underline{\underline{e}}_{y} \otimes \underline{\underline{e}}_{y} \rightarrow f^{s}\left({\underline{\underline{\sigma^{s}}}}^{s}=0\right.
\end{aligned}
$$

that is:

$$
\underline{\underline{\Sigma}}=(1-\eta) k \sqrt{3} \underline{e}_{y} \otimes \underline{e}_{y}
$$

which therefore complies with the macroscopic strength condition. The corresponding normal and shear stresses on a facet are given by (22):

$$
\Sigma_{n}(\alpha)=(1-\eta) k \frac{\sqrt{3}}{2}(1+\cos 2 \alpha) ; T(\alpha)=(1-\eta) k \frac{\sqrt{3}}{2} \sin 2 \alpha
$$

The locus of points $\left(\Sigma_{n}, T\right)(\alpha)$ for $\alpha$ ranging from $-90^{\circ}$ to $+90^{\circ}$ is therefore the circle of radius $(1-\eta) k \sqrt{3} / 2$ and centre $((1-\eta) k \sqrt{3} / 2,0)$ as shown in Figure 9. 


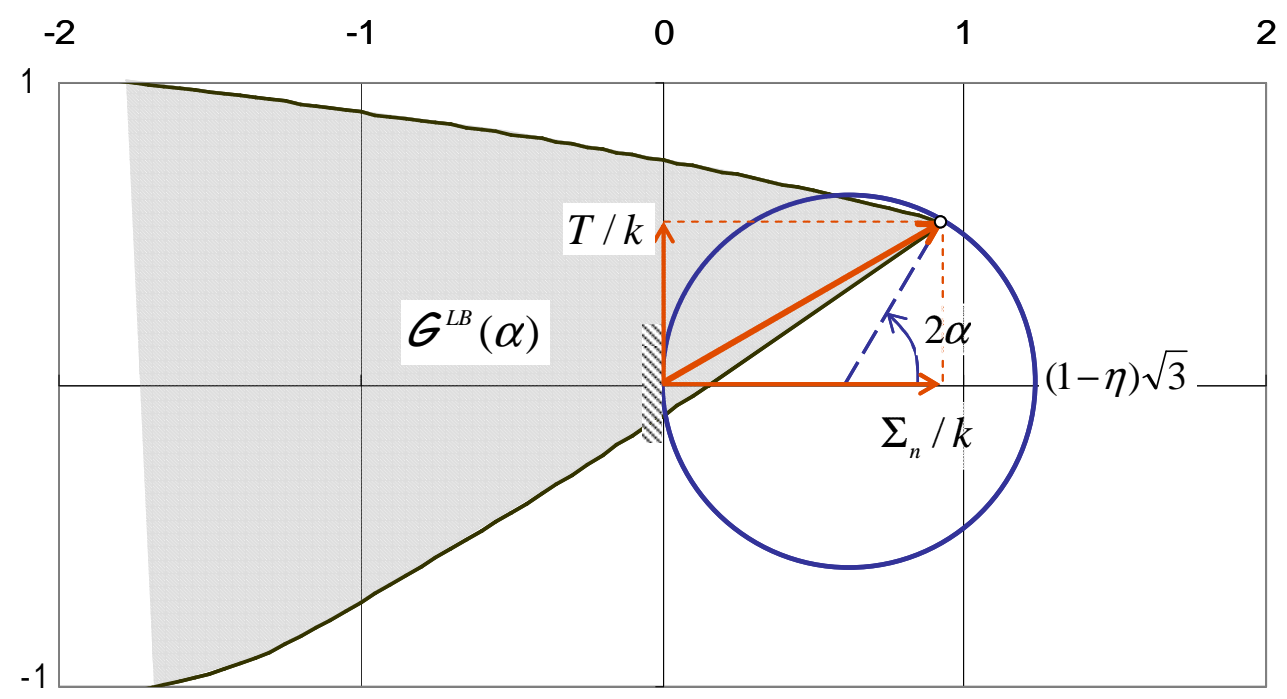

Figure 9: Locus of $\mathcal{G}^{A B}(\alpha)$ vertices in the Mohr plane

\section{An improved evaluation of the macroscopic strength criterion}

As it can be observed from Figure 5, the previously obtained lower bound approximation predicts that the strength of stone column reinforced soil subject for instance to a uniaxial tensile stress along the $O x$-direction, would be equal to zero, and more generally that the reinforced soil could not withstand any tensile stress component along this direction $\left(\Sigma_{x x} \leq 0\right)$. This appears to clearly underestimate the actual strength of the reinforced soil, since it is to be expected that, even in the absence of any reinforcing column, the surrounding cohesive soil alone would offer some resistance to such a solicitation. The objective of the present section is to derive a significantly improved estimate for the reinforced soil macroscopic strength by resorting to a numerical approach to the problem.

\subsection{Macroscopic yield surface as a result of an elastoplastic procedure}

The determination of the macroscopic strength condition and notably of its boundary yield surface in the stress space is carried out by means of a numerical elastoplastic procedure performed on the reinforced soil unit cell $C$, leading to the evaluation of limit loads along prescribed loading paths. More precisely, the unit cell is subject to a plane strain controlled radial loading path. This means that a macroscopic strain of the form: 


$$
\underline{\underline{\epsilon}}(t)=\lambda(t) \underline{\underline{\Delta}} \text { with } \underline{\underline{\Delta}}(\gamma, \delta)=\left(\begin{array}{ccc}
\cos \gamma \sin \delta & \cos \delta & 0 \\
\cos \delta & \sin \gamma \sin \delta & 0 \\
0 & 0 & 0
\end{array}\right)
$$

Is prescribed to the unit cell, where $\lambda(t)$ is a scalar multiplier increased from zero to its maximum value corresponding to the limit load, while angles $\gamma$ and $\delta$ specify the orientation of the radial loading in the space of plane strains in the $O x y$-plane (Figure 10).
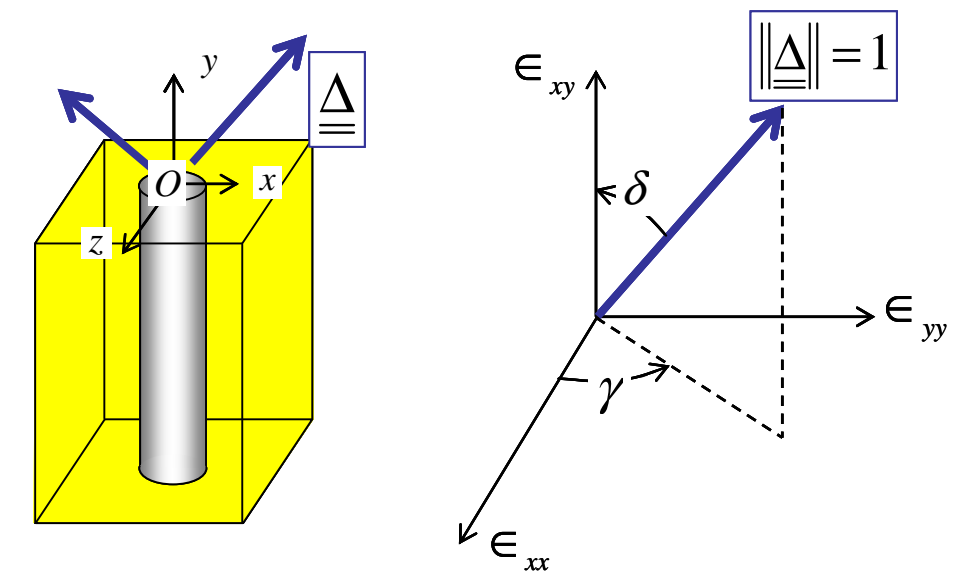

Figure 10: Angular parameterization of the plane strain controlled radial loading path of the unit cell

According to the periodic homogenization method implemented in the context of an elastoplastic behaviour (Suquet, 1985; Abdelkrim and de Buhan, 2007), the solution of the elastoplastic auxiliary problem consists in finding at each time of the loading path:

a) a velocity field defined up to a rigid body motion by:

$$
\forall \underline{\xi} \in C: \underline{\dot{u}}(\underline{\xi})=\dot{\lambda} \underline{\underline{\Delta}} \cdot \underline{\xi}+\underline{\dot{y}}(\underline{\xi})
$$

where $\underline{\dot{v}}(\underline{\xi})$ is a periodic fluctuation, so that:

$$
\langle\underline{\underline{\dot{\varepsilon}}}(\underline{\dot{\underline{v}}})\rangle=0 \rightarrow\langle\underline{\underline{\dot{\varepsilon}}}(\underline{\dot{u}})\rangle=\dot{\grave{\lambda}} \underline{\underline{\Delta}}
$$

b) a statically (and plastically) admissible periodic stress field $\underline{\underline{\sigma}}$ associated in each point to the velocity field through the elastoplastic constitutive behaviour of the material located in this point; the macroscopic stress defined by (11) represents the response of the unit cell to the previously defined strain loading path:

$$
t \in[0, T]: \dot{\underline{\epsilon}}(t)=\dot{\lambda}(t) \underline{\underline{\Delta}} \rightarrow\{\underline{\dot{u}}(t), \underline{\underline{\dot{\sigma}}}(t)\} \rightarrow \underline{\underline{\dot{\Sigma}}}(t)=\langle\underline{\underline{\dot{\sigma}}}(t)\rangle
$$




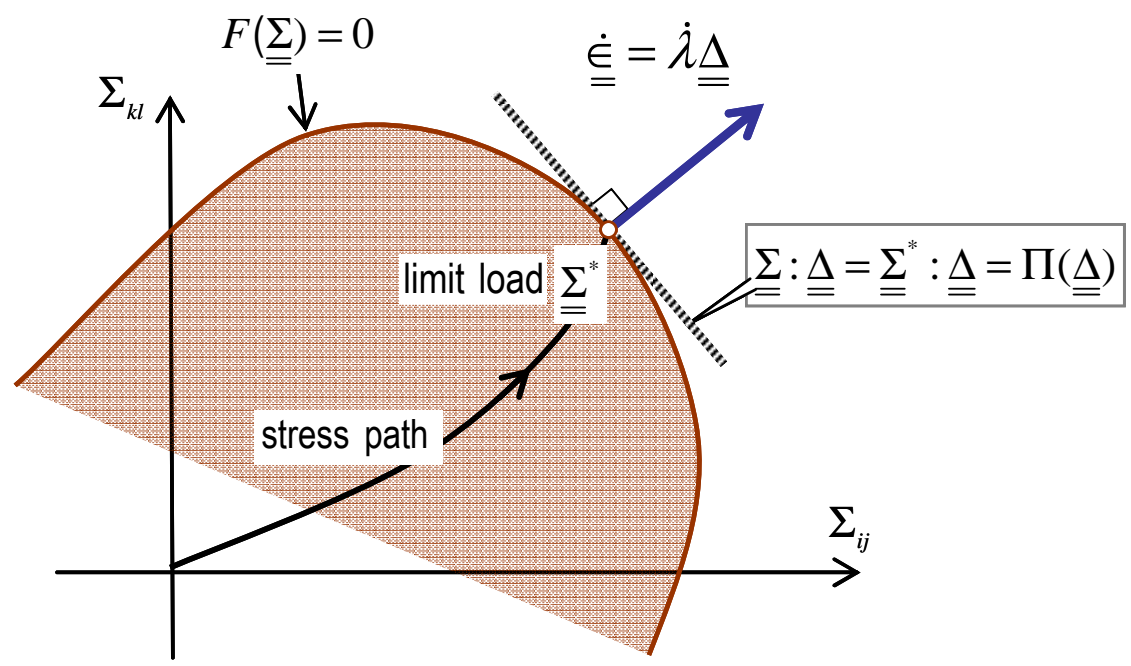

Figure 11. Elastoplastic stress response to a radial strain controlled loading and associated limit load

The corresponding loading path in the space of stresses is pictured in Figure 11 with the limit load $\underline{\underline{\Sigma}}$ identified as the intersecting point with the macroscopic yield surface. Such limit loads are characterized by the occurrence of an uncontained plastic flow mechanism on the unit cell, which means that the stress field in equilibrium with $\underline{\underline{\Sigma}}^{*}$ remains constant while the load multiplier $\lambda$ can be arbitrarily increased. The associated plastic flow rule being assumed for the elastoplastic constituent materials at the microscopic scale, the macroscopic strain rate and then $\triangleq$ is outward normal to the macroscopic yield surface at point $\underline{\underline{\Sigma}}^{*}$ :

$$
\underline{\underline{\Delta}}=\dot{\chi} \frac{\partial F}{\partial \underline{\underline{\Sigma}}}\left(\underline{\underline{\Sigma}}^{*}\right), \dot{\chi} \geq 0
$$

As a direct consequence, the support function of the macroscopic yield strength condition writes:

$$
\Pi(\underline{\underline{\Delta}})=\sup _{F(\underline{\underline{\Sigma}}) \leq 0}\{\underline{\underline{\Sigma}}: \underline{\underline{\Delta}}\}=\underline{\underline{\Sigma^{*}}}: \underline{\underline{\Delta}}
$$

so that following a given radial strain loading path, characterised by its orientation $\underline{\underline{\Delta}}$, up to plastic flow failure, yields the limit load $\underline{\underline{\Sigma}}^{*}$ as well as the evaluation of the support function for the macroscopic strength condition.

It is to be noted that such a limit load may not exist for certain orientations of the prescribed macroscopic strain, that is for certain values of angles $\gamma$ and $\delta$, which means that the macroscopic yield strength domain is unbounded in these directions and the value of the support function goes to infinity. 

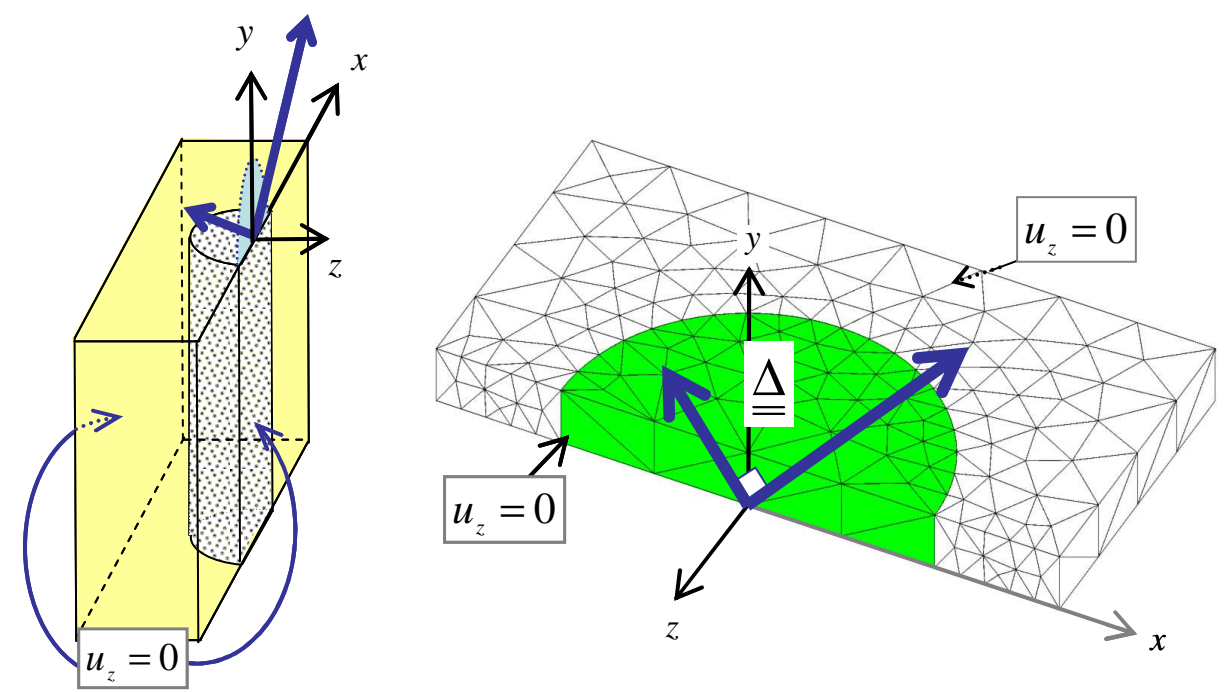

Figure 12. Finite element model adopted for solving the auxiliary problem

The elastoplastic evolution problem defined on the unit cell has been dealt with using the finite element code Cast3M (2003). Owing to the geometrical and material symmetries on the one hand, the plane strain loading configuration on the other hand, only one half of unit cell has to be considered, where zero displacements along $O z$ are prescribed on the sides parallel to the $O x y$ plane (smooth contact). Moreover, the fact that the material properties are independent of the $y$ coordinate, allows restricting the analysis to a "slice" of arbitrary thickness. This implies in practice that no particular mesh refinement is needed along this direction, as shown in Figure 12.

The loading is applied by prescribing periodicity conditions of the form (28) to the lateral sides of the model normal to the $O x$-axis, as well as to the upper and lower sides normal to the column axis $O y$. The direction of loading is modified by varying angles $\gamma$ and $\delta$ with successive increments of one degree.

It should be noted that arbitrary elastic properties can be assigned in the finite element calculations to the soil and column material since, according to a well-known result of limit analysis, the limit loads, and thus in our case the macroscopic yield surface, does not depend on those properties but exclusively on their yield strength properties (soil shear strength $k$ and column friction angle $\varphi$ ).

Figure 13 summarises the results of such numerical simulations represented in exactly the same form as that adopted in Figure 5 for the lower bound approximation to the macroscopic yield surface. A comparison with the latter approximation is given in Figure 13(b), in the form of the 
sections of the yield strength surfaces by the plane of zero shear stress. It shows a quite significant improvement of the numerical estimate, primarily in the region of tensile stresses.
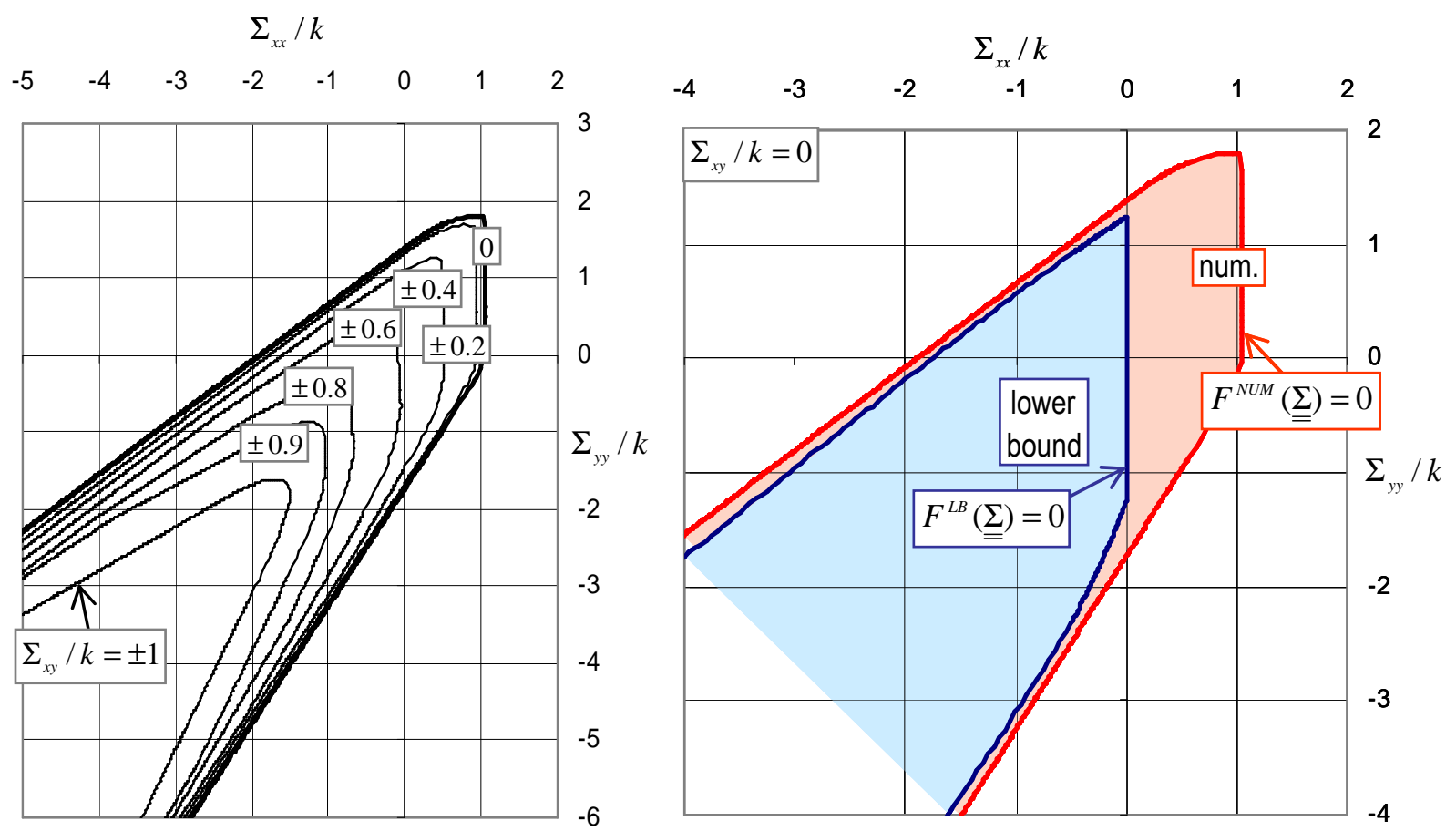

Figure 13. (a): Numerical assessment of the macroscopic yield strength surface in the stress space;

(b): comparison with the lower bound approximation

\subsection{Representation in the Mohr plane and support function for a velocity jump}

Denoting by $F^{N U M}($.$) the yield strength function associated with the numerical evaluation of the$ macroscopic strength condition (which is expected to be very close to the exact criterion), the domain of allowable stress vectors on any oriented facet is defined in the same way as for the lower bound approximation (see 3.3.):

$$
G^{N U M}(\alpha)=\left\{\left(\Sigma_{n}, T\right) ; F^{N U M}(\underline{\underline{\Sigma}}) \leq 0\right\}
$$

This domain can be alternatively characterized by means of its support function defined as:

$$
\Pi^{N U M}(\underline{n} ; \underline{V})=\sup _{\left(\Sigma_{n}, T\right)}\left\{V\left(\Sigma_{n} \sin \beta+T \cos \beta\right) ;\left(\Sigma_{n}, T\right) \in G^{N U M}(\alpha)\right\}
$$

where vector $\underline{V}$ can be interpreted as a virtual velocity jump inclined at an angle $\beta$ with the facet. The equation of the tangent to the domain $\mathcal{G}^{\mathrm{V} U M}(\alpha)$ at point $\left(\Sigma_{n}^{*}, T^{*}\right)(\beta)$ is (Figure 14):

$$
V\left(\Sigma_{n} \sin \beta+T \cos \beta\right)=\Pi^{N U M}(\underline{n} ; \underline{V})=V\left(\Sigma_{n}^{*}(\beta) \sin \beta+T^{*}(\beta) \cos \beta\right)
$$


so that $G^{V U M}(\alpha)$ may be drawn as the convex envelope of the family of straight lines (35) depending on the angular parameter $\beta$.

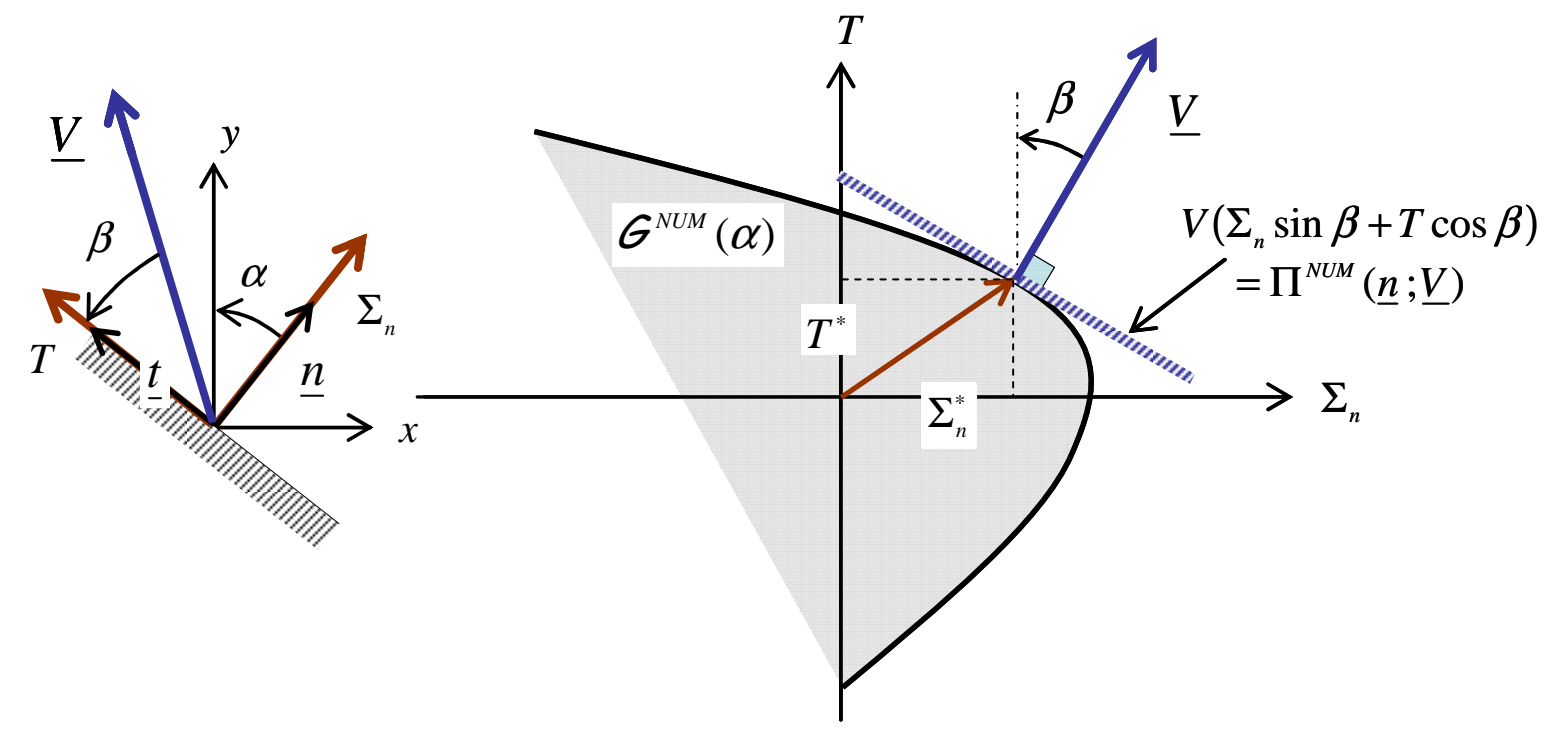

Figure 14. Geometrical interpretation of the support function of $G^{N U M}(\alpha)$

Now this support function can be directly evaluated from the previous numerical simulations. Indeed, making use of the relations (22) as well as of definition (33), it can be rewritten as:

$$
\begin{aligned}
& \Pi^{N U M}(\underline{n} ; \underline{V})=\sup _{\underline{\Sigma}}\left\{\underline{\underline{\Sigma}}:(V \sin \beta \underline{n} \otimes \underline{n}+V \cos \beta \underline{t} \otimes \underline{n}) ; F^{N U M}(\underline{\underline{\Sigma}}) \leq 0\right\} \\
& =\sup _{\underline{\underline{\Sigma}}}\left\{\underline{\underline{\Sigma}}:(\underline{V} \otimes \underline{n}) ; F^{N U M}(\underline{\underline{\Sigma}}) \leq 0\right\}=\sup _{\underline{\underline{\Sigma}}}\left\{\underline{\underline{\Sigma}}: \underline{\underline{\dot{E}}} ; F^{N U M}(\underline{\underline{\Sigma}}) \leq 0\right\}=\Pi^{N U M}(\underline{\underline{\dot{\epsilon}}})
\end{aligned}
$$

where $\underline{\underline{E}}=1 / 2(\underline{n} \otimes \underline{V}+\underline{V} \otimes \underline{n})$ is a particular macroscopic plane strain rate of the form (27) explored in the above elastoplastic numerical simulations, which depends on angles $\alpha$ and $\beta$ :

$$
\stackrel{\dot{\epsilon}}{=}(\alpha, \beta)=V\left(\begin{array}{ccc}
\cos \alpha \sin (\beta-\alpha) & 1 / 2 \cos (\beta-2 \alpha) & 0 \\
1 / 2 \cos (\beta-2 \alpha) & \sin \alpha \cos (\beta-\alpha) & 0 \\
0 & 0 & 0
\end{array}\right)
$$

Finite element elastoplastic simulations are thus carried out as follows. For each value of the facet orientation $\alpha$, ranging from $0^{\circ}$ to $90^{\circ}$, the angle $\beta$ specifying the direction of the velocity jump is varied from $0^{\circ}$ to $180^{\circ}$. Denoting by $\underline{\underline{\Sigma}}^{*}(\alpha, \beta)$ the computed value of the limit stress (if existent) along this strain controlled path, the support function is calculated as:

$$
\Pi^{N U M}(\underline{n} ; \underline{V})=\underline{\underline{\Sigma^{*}}}(\alpha, \beta): \underline{\dot{\epsilon}}
$$


and the boundary line of $G^{N U M}(\alpha)$ is then drawn as the locus of points $\left(\Sigma_{n}^{*}, T^{*}\right)(\beta)$ or as the envelope of its tangent lines (Figure 14):

$$
\left(\Sigma_{n}^{*}(\beta)-\Sigma_{n}\right) \sin \beta+\left(T^{*}(\beta)-T\right) \cos \beta=0
$$
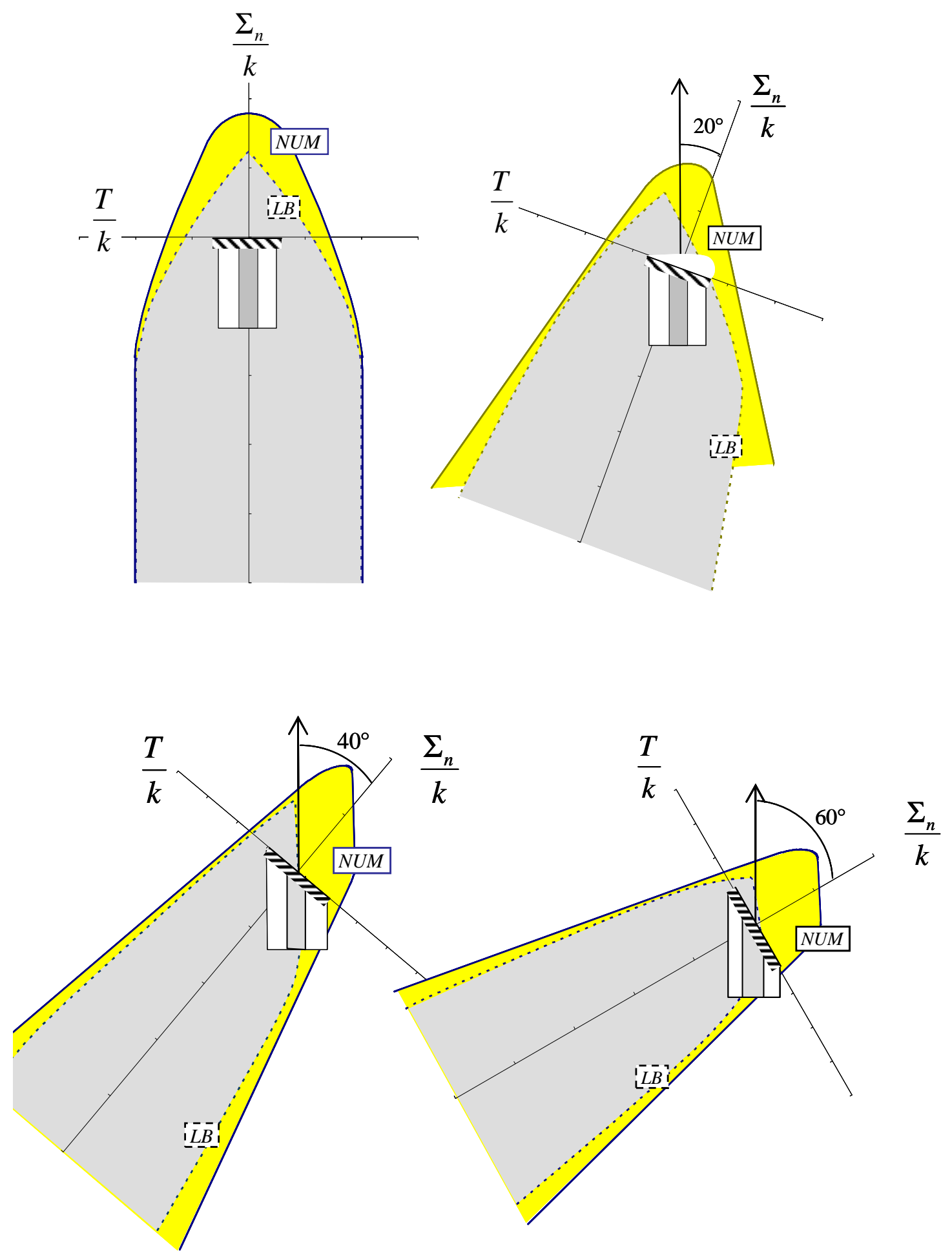


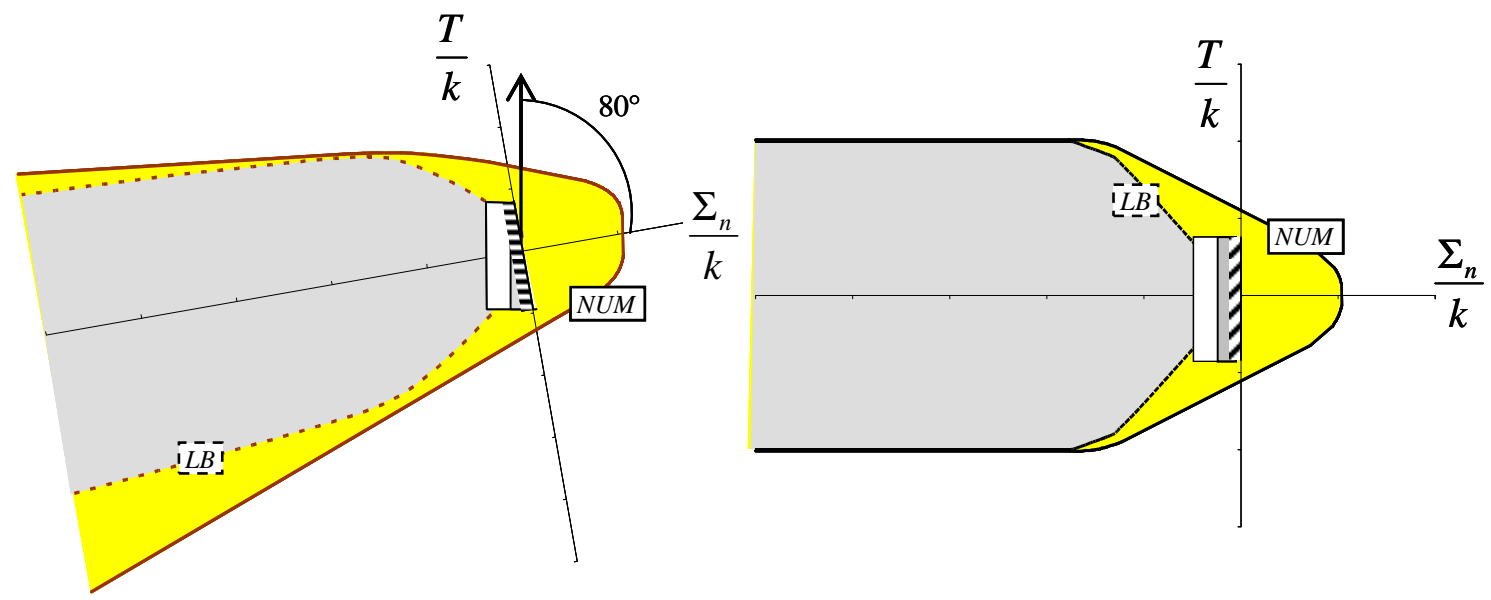

Figure 15. Representation of the macroscopic strength criterion in the Mohr plane for different facet orientations: comparison between numerical estimates and lower bound approximations

The results of this procedure are represented in Figures 15 for different values of $\alpha$, where the lower bound approximation is compared with the numerical evaluation. This confirms that the numerical procedure leads to significantly improved estimates for the actual criterion, specifically in the range of tensile normal stresses. Figure 16 where all numerical results are gathered in the same Mohr plane, shows the evolution of the yield strength curve as a function of the facet orientation, which is a clear indicator of the reinforced soil strength anisotropy.

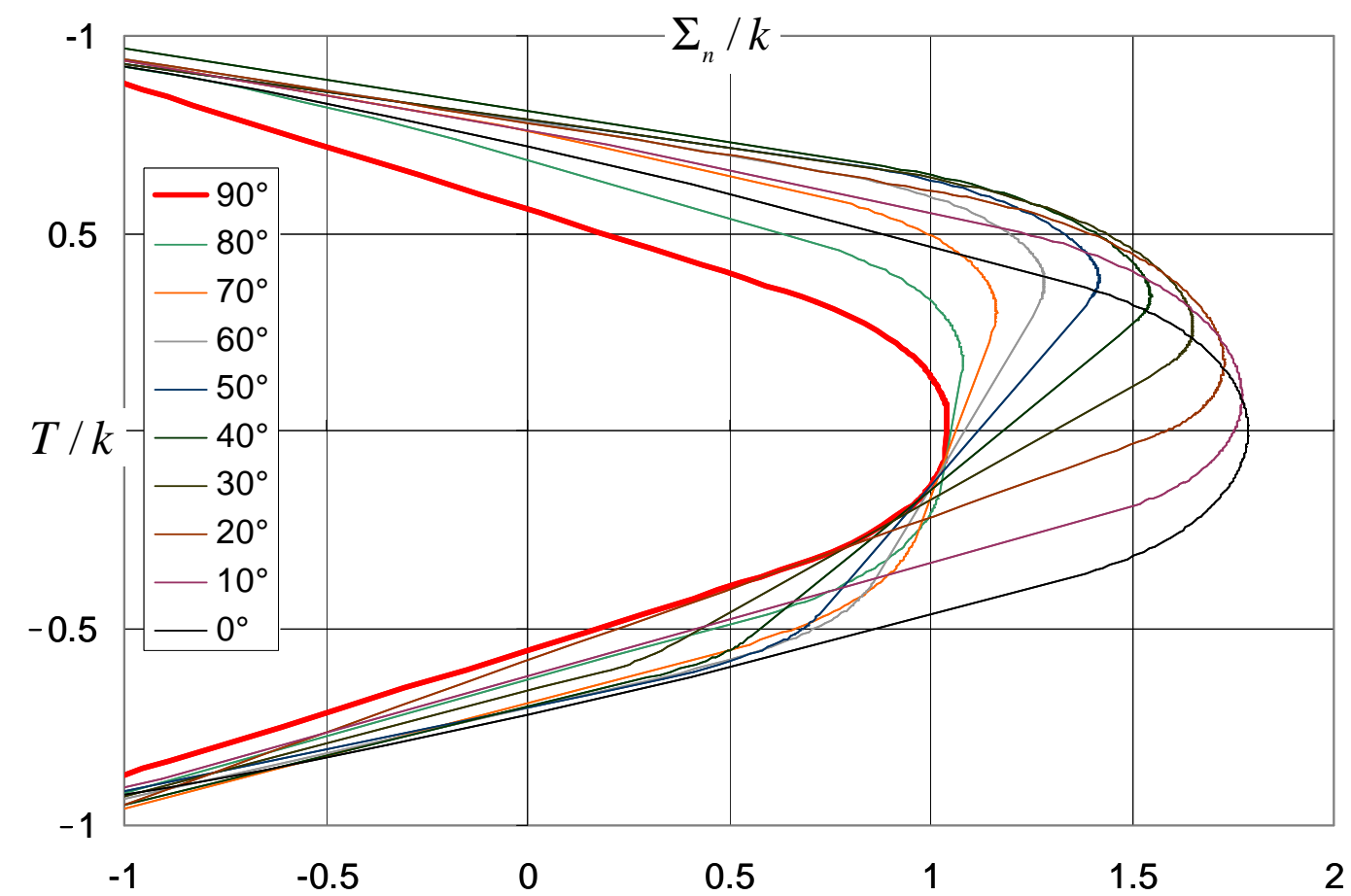

Figure 16. Macroscopic yield strength curves in the Mohr plane for different facet orientations 
This inherent strength anisotropy is further illustrated from calculating the value of the support function relative to a velocity discontinuity, which may be written as:

$$
\Pi^{N U M}(\underline{n} ; \underline{V})=k V \pi^{n u m}(\alpha, \beta)
$$

where $\pi^{n u m}$ is a non dimensional function of angular parameters $\alpha$ and $\beta$, represented in Figure 17.

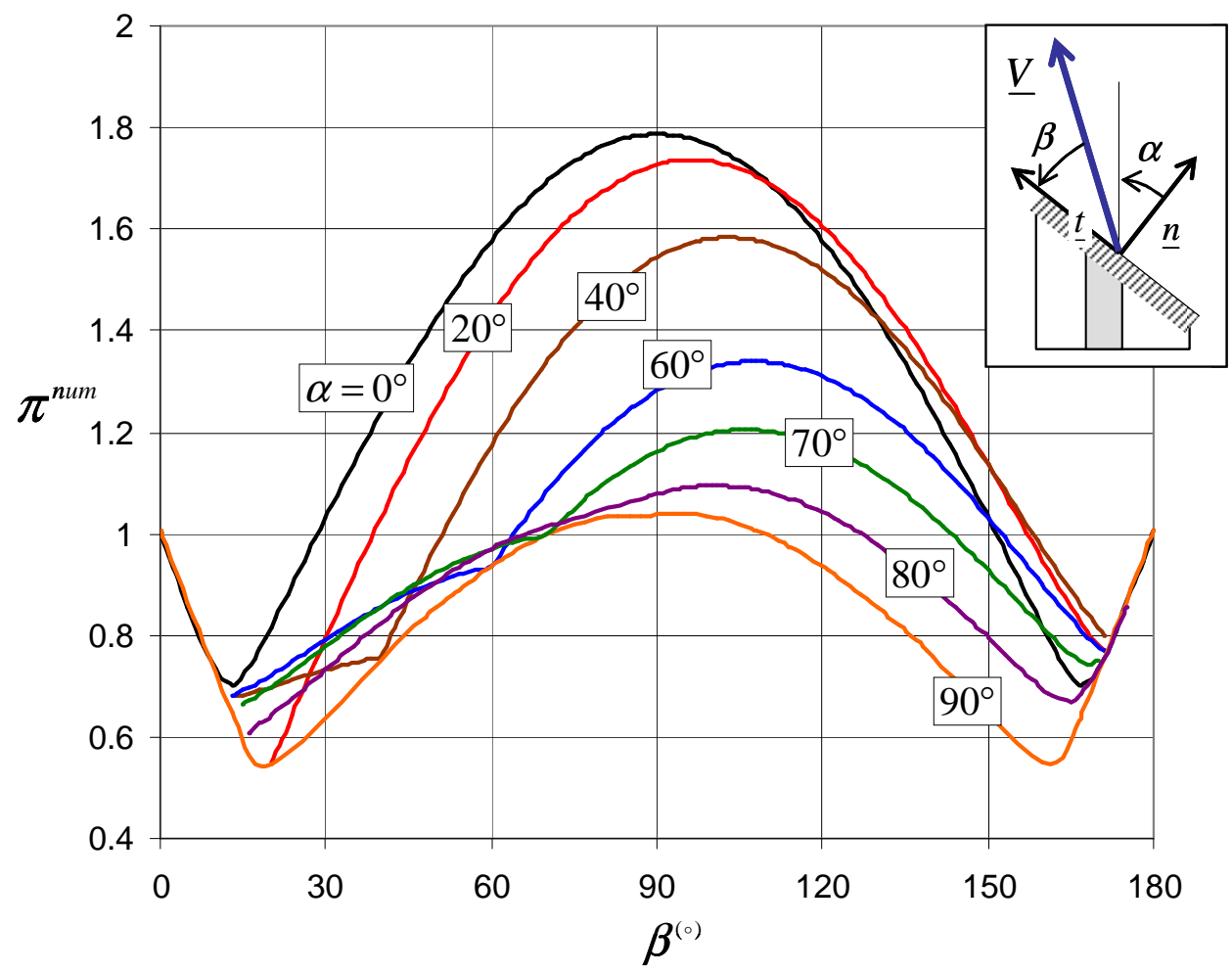

Figure 17. Non dimensional value of the support function for a velocity jump across differently oriented facets

\section{Application to the failure design of a stone column reinforced foundation}

\subsection{Problem statement}

As a first illustrative application of the upper bound kinematic approach of yield design, using the previously obtained numerical estimate of the reinforced soil macroscopic strength domain, the following problem is considered. A soil layer of thickness $H=20 \mathrm{~m}$ and horizontal extension $L=80 \mathrm{~m}$ is subject to vertical loading applied though a rigid strip footing of width $B=5 \mathrm{~m}$ as shown in Figure 18(a). In order to enhance the load bearing capacity of the soil, a group of floating stone 
columns of length $l=15 \mathrm{~m}$ placed beneath the footing has been incorporated into the soil following a regular arrangement.

The native soil is a soft clay obeying a von Mises criterion with $k$ as strength parameter, while the granular ballast of the reinforcing columns obeys a Drucker-Prager strength condition with a typical value of the friction angle equal to $\varphi=35^{\circ}$. The reinforcement volume fraction is equal to $\eta \cong 28 \%$ which corresponds to a column radius $\rho$ equal to 0.3 the spacing $s$ between adjacent columns. For the sake of simplicity, the role of gravity is omitted in the subsequent analysis.

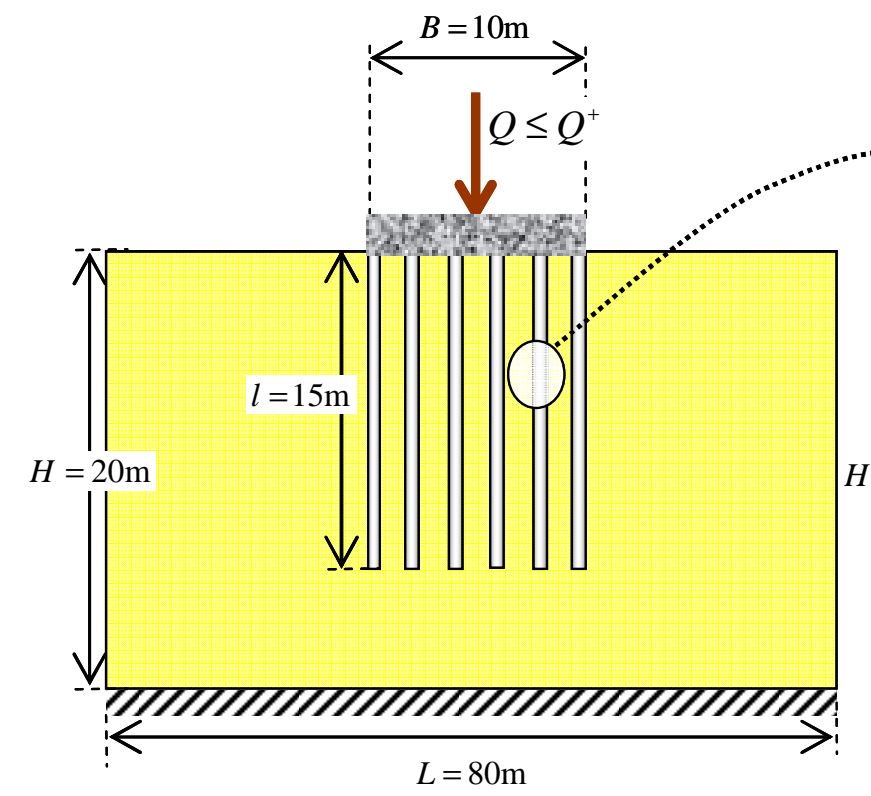

(a)

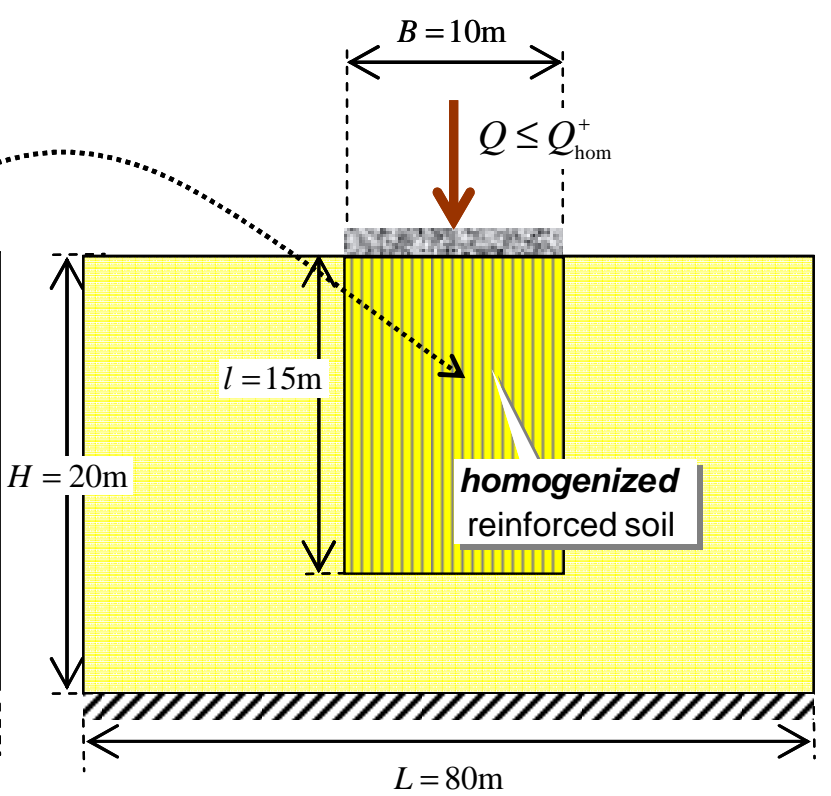

$(b)$

Figure 18. Ultimate bearing capacity analysis of a stone column reinforced foundation: $(a)$ initial and $(b)$ homogenized problems

Denoting by $Q$ the line density of load applied along the footing axis, the ultimate load bearing capacity of the foundation may be expressed as a function of a non dimensional parameter $\varepsilon$ defined as the ratio between the columns spacing $s$ and the footing width $B$ :

$$
Q \leq Q^{+}(\varepsilon) \text { with } \varepsilon=s / B
$$

with all the other parameters being kept fixed. The yield design homogenization method (Suquet, 1985; de Buhan, 1986) is based upon the following convergence property:

$$
\lim _{\varepsilon \rightarrow 0} Q^{+}(\varepsilon)=Q_{\mathrm{hom}}^{+}
$$


$Q_{\mathrm{hom}}^{+}$represents the ultimate bearing capacity of the homogenized problem (Figure 18(b)), where the stone column reinforced ground has been replaced by an equivalent homogenous medium obeying the previously determined macroscopic strength criterion. Unlike the initial problem, this homogenized problem can be dealt with as a yield design plane strain problem

\subsection{Analysis by the upper bound kinematic approach}

An upper bound estimate for $Q_{\text {hom }}^{+}$is now searched by means of the yield design kinematic approach using the very simple failure mechanism of Figure 19. This mechanism is made of two rectangular triangular blocks, involving three velocity discontinuity lines. The triangular block 1 located under the footing, characterized by angle $\alpha_{1}$ is given a velocity $\underline{U}_{1}$ inducing a velocity jump inclined at angle $\beta_{1}$ with respect to the lower discontinuity line $A C$ across the homogenized reinforced soil. The second adjacent triangular block 2 characterized by angle $\alpha_{2}$ is given a uniform translation of vector $\underline{U}_{2}$. The corresponding lower discontinuity line $C D$ is located in the purely cohesive native soil, so that the velocity jump must remain tangential in order to yield a finite value of the support function (see Eq. (5)).

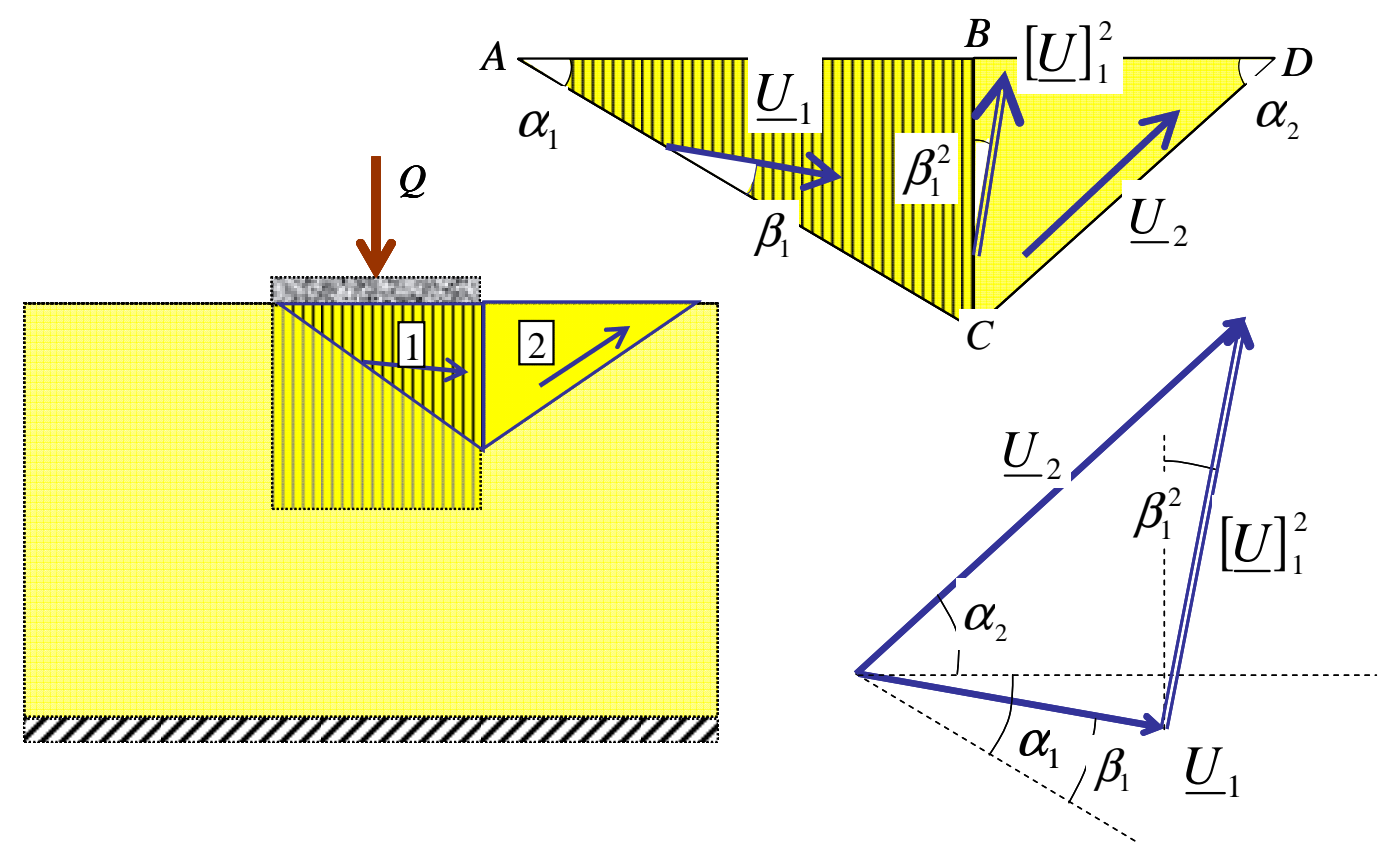

Figure 19. Rigid block failure mechanism used in the yield design kinematic approach

Such a mechanism involves a vertical discontinuity line $B C$ between the two blocks with a velocity jump equal to:

$$
[\underline{U}]_{1}^{2}=\underline{U}_{2}-\underline{U}_{1}
$$


and inclined at an angle $\beta_{1}^{2}$ with the vertical.

It turns out that the mechanism under consideration may be entirely defined by four angular parameters $\left(\alpha_{1}, \alpha_{2}, \beta_{1}, \beta_{1}^{2}\right)$ along with the norm $U_{1}$ of the velocity of block 1 . The hodograph of velocities drawn in Figure 19, makes it then possible to calculate the norms of the velocity of block 2 as well as of that of the inter block velocity jump, through the following geometrical relationships:

$$
U_{2}=U_{1} \frac{\cos \left(\alpha_{1}-\beta_{1}-\beta_{1}^{2}\right)}{\cos \left(\alpha_{2}+\beta_{1}^{2}\right)} ;[U]_{1}^{2}=U_{1} \frac{\sin \left(\alpha_{1}+\alpha_{2}-\beta_{1}\right)}{\cos \left(\alpha_{2}+\beta_{1}^{2}\right)}
$$

The virtual work of external forces, calculated per unit length along the footing axis, in this failure mechanism may be expressed as:

$$
W_{e}\left(U_{1}, \alpha_{1}, \beta_{1}\right)=Q U_{1} \sin \left(\alpha_{1}-\beta_{1}\right)
$$

On the other hand, since only velocity discontinuities are involved in this mechanism, the maximum resisting work writes:

$$
\begin{aligned}
W_{m r}\left(\underline{U}_{1}, \underline{U}_{2}\right) & =\int_{A C} \pi\left(\underline{n}_{A C}, \underline{U}_{1}\right) \mathrm{d} s+\int_{B C} \pi\left(\underline{n}_{B C},[\underline{U}]_{1}^{2}\right) \mathrm{d} s+\int_{C D} \pi\left(\underline{n}_{C D}, \underline{U}_{2}\right) \mathrm{d} s \\
& =A C \pi\left(\underline{n}_{A C}, \underline{U}_{1}\right)+B C \pi\left(\underline{n}_{B C},[\underline{U}]_{1}^{2}\right)+C D \pi\left(\underline{n}_{C D}, \underline{U}_{2}\right)
\end{aligned}
$$

with

$$
A C=B / \cos \alpha_{1}, B C=B \tan \alpha_{1} \text { and } C D=B \tan \alpha_{1} / \sin \alpha_{2}
$$

where $\underline{n}_{I J}$ denotes the normal unit vector to the discontinuity line $I J$. The different support functions are calculated as follows.

- Along the segment $C D$ located in the soil where the velocity jump $\underline{U}_{2}$ is tangential:

$$
\pi\left(\underline{n}_{C D}, \underline{U}_{2}\right)=k U_{2}=k U_{1} \frac{\cos \left(\alpha_{1}-\beta_{1}-\beta_{1}^{2}\right)}{\cos \left(\alpha_{2}+\beta_{1}^{2}\right)}
$$

- Along the segment $A C$ located in the homogenized reinforced zone, the support function is given by (40):

$$
\pi\left(\underline{n}_{A C}, \underline{U}_{1}\right)=k U_{1} \pi^{n u m}\left(\alpha_{1}, \beta_{1}\right)
$$

- Finally, the third discontinuity line $B C$ being located at the interface between the reinforced zone and the soil, the support function is: 


$$
\pi\left(\underline{n}_{B C},[\underline{U}]_{1}^{2}\right)=k[U]_{1}^{2} \pi^{n u m}\left(90^{\circ}, \beta_{1}^{2}\right)=k U_{1} \frac{\sin \left(\alpha_{1}+\alpha_{2}-\beta_{1}\right)}{\cos \left(\alpha_{2}+\beta_{1}^{2}\right)} \pi^{n u m}\left(90^{\circ}, \beta_{1}^{2}\right)
$$

Putting Eqs. (46) to (49) together, one finally gets:

$$
W_{m r}\left(U_{1}, \alpha_{1}, \alpha_{2}, \beta_{1}, \beta_{1}^{2}\right)=B k U_{1} w_{m r}\left(\alpha_{1}, \alpha_{2}, \beta_{1}, \beta_{1}^{2}\right)
$$

with

$$
\begin{aligned}
& w_{m r}\left(\alpha_{1}, \alpha_{2}, \beta_{1}, \beta_{1}^{2}\right)= \\
& \frac{\tan \alpha_{1} \cos \left(\alpha_{1}-\beta_{1}-\beta_{1}^{2}\right)}{\sin \alpha_{2} \cos \left(\alpha_{2}+\beta_{1}^{2}\right)}+\frac{1}{\cos \alpha_{1}} \pi^{n u m}\left(\alpha_{1}, \beta_{1}\right)+\tan \alpha_{1} \frac{\cos \left(\alpha_{1}+\alpha_{2}-\beta_{1}\right)}{\cos \left(\alpha_{2}+\beta_{1}^{2}\right)} \pi^{n u m}\left(90^{\circ}, \beta_{1}^{2}\right)
\end{aligned}
$$

The application of the kinematic approach of yield design to the homogenized problem states that a necessary condition for the loading to remain below the ultimate load bearing capacity writes:

$$
Q \leq Q_{\mathrm{hom}}^{+} \Rightarrow \forall \underline{U}, W_{e}(\underline{U}) \leq W_{m r}(\underline{U})
$$

that is on account of (45) and (50) and after simplification by $U_{1}$ :

$$
\forall\left(\alpha_{1}, \alpha_{2}, \beta_{1}, \beta_{1}^{2}\right), \quad Q_{\mathrm{hom}}^{+} \leq k B \frac{w_{m r}\left(\alpha_{1}, \alpha_{2}, \beta_{1}, \beta_{1}^{2}\right)}{\sin \left(\alpha_{1}-\beta_{1}\right)}
$$

The minimization of the so obtained upper bound with respect to the angular parameters $\alpha_{1}$, $\alpha_{2}, \beta_{1}$ and $\beta_{1}^{2}$ is then performed numerically using the results of the procedure described in the previous section for the evaluation of function $\pi^{n u m}$. It thus leads to the following best upper bound to be derived from the considered family of failure mechanism:

$$
Q_{\text {hom }}^{+} \leq 7.15 k B
$$

which corresponds to the following set of angular parameters:

$$
\alpha_{1}=46^{\circ}, \alpha_{2}=35^{\circ}, \beta_{1}=12^{\circ}, \beta_{1}^{2}=0^{\circ}
$$

It is to be noted that the optimized mechanism is associated with a tangential velocity jump $\beta_{1}^{2}=0^{\circ}$ across the discontinuity line $B C$ separating the two translating blocks. This means that the optimized upper bound would have been exactly the same if the $B C$ had been located in the soil and non in the reinforced zone. 
The above upper bound estimate (54) is worth being compared with that obtained for a non reinforced soil foundation, using the same class of two blocks failure mechanism. Indeed, in such a case the upper bound estimate is:

$$
Q^{+} \leq 6 k B
$$

so that an increase of the load bearing capacity almost equal to $20 \%$ might be expected from the reinforcement by stone columns.

\section{Conclusion and perspectives}

The feasibility of a yield design homogenization method aimed at overcoming the difficulties inherent in a direct stability analysis of stone column reinforced soil structures, has been clearly demonstrated in this contribution on a simple illustrative example. This method relies upon the preliminary formulation of a macroscopic strength criterion for the composite reinforced soil regarded as an equivalent homogeneous, but anisotropic, continuum.

The determination of this macroscopic strength condition is derived from the solution of a specific yield design boundary value problem attached to the reinforced soil unit representative cell. A first qualitative assessment of this criterion is obtained from a lower bound approximation based on the consideration of piecewise constant stress fields defined on the unit cell. As a novel result, an improved and much more accurate yield locus has then been drawn from elastoplastic numerical simulations along radial strain controlled loading paths followed up to failure.

The resulting strength domain represented in the space of stresses shows that the stone column reinforced soil exhibits both cohesive and frictional properties, with a marked anisotropy due to the preferential orientation of the reinforcing columns. This is further illustrated by drawing the domain of allowable stresses in the Mohr plane for different facet orientations, which is perfectly equivalent to calculating the support function relative to a velocity jump in the homogenized reinforced soil. The tabulated numerical values obtained for the latter function make it thus possible to perform the kinematic approach of yield design on the homogenized problem, leading to an upper bound estimate for the reinforced foundation ultimate bearing capacity computed from considering a very simple two blocks failure mechanism.

The extension of the proposed yield design homogenization method to more complex failure mechanisms likely to produce improved, and thus more reliable, stability analyses of stone column reinforced soil structures highly depends on the fact that the macroscopic strength condition should remain easy to manipulate. Simplified closed form expressions are therefore to be preferred 
to the quite cumbersome tabulated numerical values used so far. This sets the agenda of future research works aimed at turning the homogenization method into an innovative engineering design procedure. 


\section{References}

Abdelkrim M. , de Buhan P. (2007). An elastoplastic homogenization procedure for predicting the settlement of a foundation on a soil reinforced by columns. Eur. J. Mech., A/Solids, 26, pp. 736-757.

Abdi R., de Buhan P., Pastor J. (1994). Calculation of the critical height of a homogenized reinforced soil wall: a numerical approach. Int. J. Num. Anal. Meth. Geomech., vol. 18, pp. 485-505.

Broms B.G. (1982). Lime columns in theory and practice. Proc. Int. Conf. of Soil Mechanics, Mexico, pp. 149-165.

Cast3M (2003) http://www-cast3m.cea.fr

de Buhan P. (1986). A fundamental approach to the yield design of reinforced soil structures (in French), Thèse d'Etat, Paris VI.

de Buhan P., Salençon J. (1987). Yield strength of reinforced soils as anisotropic media. IUTAM Symposium on Yielding, Damage and Failure of Anisotropic Solids, Grenoble, Ed. J.P. Boehler, Mech. Eng. Publ., London, pp. 791-803.

de Buhan P., Mangiavacchi R., Nova R., Pellegrini G., Salençon J. (1989). Yield design of reinforced earth walls by a homogenization method. Géotechnique, 39, n², pp. 189-201.

de Buhan P., Taliercio A. (1991). A homogenization approach to the yield strength of composite materials. Eur. J. Mech., A/Solids, 10(2), pp. 129-50.

Jellali B., Bouassida, M. and de Buhan, P. (2005). A homogenization method for estimating the bearing capacity of soils reinforced by columns. Int. Numer Anal Meth Geomech, Vol 29, pp 116.

Jellali B., Bouassida M., de Buhan P. (2007). A homogenization approach to estimate the ultimate bearing capacity of a stone column-reinforced foundation. Int. J. Geotech. Eng., 1, 9, pp. 6169.

Jellali B., Bouassida, M. and de Buhan, P. (2011). Stability analysis of an embankment resting upon a column-reinforced soil. Int. Numer Anal Meth Geomech, Vol 35, pp 1243-1256.

McLaughlin P.V. (1972). Plastic limit behavior of filament reinforced materials. Int. J. Sol. Struct., 8, pp. 1299-1318.

Majamdar S., McLaughlin P.V. (1975). Effects of phase geometry and volume fraction on the plane stress limit analysis of a unidirectional fiber-reinforced composite. Int. J. Sol. Struct., 11, pp. 777-791. 
Michalowski R.L., Zhao A. (1995). A continuum vs. structural approach to stability of reinforced soil. J. Geotech. Eng., ASCE, 121(2), pp. 152-162.

Michalowski R.L. (1997). Stability of uniformly reinforced slopes. J. Geotech. Eng., vol. 123, n6, pp. 546-556.

Priebe H. (1995). The design of vibroreplacement. Ground Engineering, December, pp. 31-37.

di Prisco C. Nova R. (1993). A constitutive model for soil reinforced by continuous threads. Geotext. Geomembr., 12, pp. 161-178.

Salençon J. (1990). An introduction of the yield design theory and its application to soil mechanics. Eur. J. Mech. A/ Solids, 9, n5, pp. 477-500.

Sawicki A. (1983). Plastic limit behavior of reinforced earth. J. Geotech. Eng., ASCE, 129(7), pp. 1000-1005.

Sawicki A., Lesniewska D. (1989). Limit analysis of cohesive slopes reinforced with geotextiles. Comp. Geotech., 7, pp. 53-66.

Suquet P. (1985). Elements of homogenization for inelastic solid mechanics. In: CISM Lecture Notes, $\mathrm{n}^{\circ} 272$, "Homogenization Techniques for Composite Media", Springer-Verlag, pp. 193278. 